\title{
A Langevin Description for Driven Granular Gases
}

\author{
P. Maynar and M. I. García de Soria * \\ Física Teórica, Universidad de Sevilla, Apartado de Correos 1065, E-41080, Sevilla, Spain
}

\begin{abstract}
The study of the fluctuations in the steady state of a heated granular system is reviewed. A Boltzmann-Langevin description can be built requiring consistency with the equations for the one- and two-particle correlation functions. From the Boltzmann-Langevin equation, Langevin equations for the total energy and the transverse velocity field are derived. The existence of a fluctuation-dissipation relation for the transverse velocity field is also studied.
\end{abstract}

Key words: kinetic theory, Boltzmann equation, granular gases, hydrodynamics AMS subject classification: 76P05, 76T25, 82B40, 82C40, 82D05

\section{Introduction}

A granular system is characterized by a continuous loss of energy in collisions. The simplest model that we can consider to describe this kind of systems is an assembly of smooth hard spheres (or disks in two dimensions) that collide inelastically, in such a way that part of the kinetic energy is dissipated in the encounter. The collision is characterized by a constant coefficient of normal restitution $[16,21]$. The rich phenomenology that has been found for this kind of systems makes them of particular interest from both a fundamental and an applied point of view. In the dilute limit, it has been shown numerically that for a wide class of initial conditions an isolated granular gas reaches a homogeneous state, the so-called homogeneous cooling state (HCS), in which all the time dependence of the one-particle distribution function is embedded in the granular temperature which is defined as the second velocity moment of the distribution [17, 18]. This state, whose properties have been extensively studied in the literature, plays, for inelastic gases, a role similar to that of the equilibrium state for molecular gases. Nevertheless, from an experimental point of view,

*Corresponding author. E-mail: gsoria@us.es 
it is not obvious to probe such a regime $[19,25]$. However, it is possible to obtain a non-equilibrium stationary state by supplying energy to the system in such a way to compensate the dissipation due to the inelasticity of collisions. We can think of several different procedures to thermalise the system. An inhomogeneous stationary state can be reached if the energy is injected by a moving boundary such as a vibrating piston [8]. To obtain a spatially homogeneous stationary state, we can consider a mechanism coupling the velocity of each particle to a white noise, the so-called stochastic thermostat $[10,11,12,15,22,23,24,27,30,31,32,33]$. For this kind of forcing, which is relevant for some two-dimensional experimental configurations with a rough vibrating piston [26], the system reaches a homogeneous stationary state after a transient regime. The Boltzmann equation for the steady state can be written and the distribution function evaluated approximately for thermal velocities. For large values of the modulus of the velocity, an exponential behaviour is found. An expression for the stationary temperature can be obtained as a function of the strength of the noise and of the inelasticity of collisions [30].

The aim of this paper is to review some recent results about the fluctuations for a system subject to the stochastic thermostat $[14,20]$ generalizing the theory to the study of dynamical properties. The study of the fluctuations of granular gases has been addressed for a system in the homogeneous cooling state [7]. The starting point for this study was the equations for the correlation functions obtained from an extension of the BBGKY hierarchy for granular gases. Later, following the ideas of Zwanzig [2], a Langevin equation for the distribution function was introduced, in which the properties of the noise of the Boltzmann-Langevin equation were identified requiring consistency with the equations for the correlations [6]. A similar method will be applied to the study of heated granular gases, identifying the Boltzmann-Langevin equation for the fluctuating distribution function close to the stationary state. Once the fundamental properties of the noise of the equations are calculated, we focus on the derivation of Langevin equations for the total energy and the transverse velocity. From these equations, the one-time and two-time correlation functions are evaluated. We pay special attention to the study of the transverse velocity remarking the differences with the elastic case (where we have a fluctuation-dissipation relation) and with the undriven inelastic case. We note that in previous studies fluctuating equations for the hydrodynamic fields were introduced phenomenologically, expecting to provide a valid description, at least, for small values of the inelasticity [31]. The Boltzmann-Langevin description, as we will show, is valid for all the range of values of the inelasticity, and allows us to address the problem of finding a fluctuating hydrodynamic description without the limitation of small inelasticity.

The remainder of the paper is organised as follows. In Sec. 2. we recall some general results for a granular gas heated by a stochastic thermostat. Sec. 3. is devoted to the non-fluctuating hydrodynamic equations. In Sec. 4., the Boltzmann-Langevin description is presented and it is finally applied to the derivation of the Langevin equations for the energy and the transverse velocity field in Sec. 5. and 6., respectively. 


\section{Kinetic theory description of heated granular gases}

The system considered is a dilute gas of $N$ smooth inelastic hard particles of mass $m$ and diameter $\sigma$. The position and velocity of the $i$ th particle at time $t$ will be denoted by $\mathbf{R}_{i}(t)$ and $\mathbf{V}_{i}(t)$, respectively. The effect of a collision between two particles $i$ and $j$ is to instantaneously modify their velocities according to the collision rule

$$
\begin{aligned}
\mathbf{V}_{i}^{\prime} & =\mathbf{V}_{i}-\frac{1+\alpha}{2}\left(\hat{\boldsymbol{\sigma}} \cdot \mathbf{V}_{i j}\right) \hat{\boldsymbol{\sigma}} \\
\mathbf{V}_{j}^{\prime} & =\mathbf{V}_{j}+\frac{1+\alpha}{2}\left(\hat{\boldsymbol{\sigma}} \cdot \mathbf{V}_{i j}\right) \hat{\boldsymbol{\sigma}}
\end{aligned}
$$

where $\mathbf{V}_{i j} \equiv \mathbf{V}_{i}-\mathbf{V}_{j}$ is the relative velocity, $\hat{\boldsymbol{\sigma}}$ is the unit vector pointing from the center of particle $j$ to the center of particle $i$ at contact, and $\alpha$ is the coefficient of normal restitution. It is defined in the interval $0<\alpha \leq 1$ and it will be considered here as a constant, independent of the relative velocity. Between collisions, the system is heated uniformly by adding a random velocity to the velocity of each particle at equal times. The driving is implemented in such a way that the time between random kicks is small compared to the mean free time. Then, between collisions, the velocities of the particles undergo a large number of kicks due to the thermostat. In addition, we will assume that the "jump moments" of the velocities of the particles verify

$$
\begin{array}{r}
B_{i j, \beta \gamma} \equiv \lim _{\Delta t \rightarrow 0} \frac{\left\langle\Delta V_{i, \beta} \Delta V_{j, \gamma}\right\rangle_{\text {noise }}}{\Delta t}=\xi_{0}^{2} \delta_{i j} \delta_{\beta \gamma}+\frac{\xi_{0}^{2}}{N}\left(\delta_{i j}-1\right) \delta_{\beta \gamma}, \\
i, j=1, \ldots, N \quad \text { and } \quad \beta, \gamma=1, \ldots, d,
\end{array}
$$

where we have introduced $\Delta V_{i, \beta} \equiv V_{i, \beta}(t+\Delta t)-V_{i, \beta}(t), V_{i, \beta}(t)$ being the $\beta$ component of the velocity of particle $i$ at time $t$. We have also introduced the strength of the noise, $\xi_{0}$, and $\langle\ldots\rangle_{n o i s e}$, which denotes average over different realizations of the noise. The non-diagonal terms (corresponding to $i \neq j$ and $\beta=\gamma$ ) are necessary in order to conserve the total momentum [14].

\subsection{Kinetic Equations}

Given a trajectory of the system, microscopic densities in phase space, $F_{s}\left(x_{1}, \ldots, x_{s}, t\right)$, at time $t$ are defined by

$$
\begin{gathered}
F_{1}\left(x_{1}, t\right)=\sum_{i=1}^{N} \delta\left[x_{1}-X_{i}(t)\right], \\
F_{2}\left(x_{1}, x_{2}, t\right)=\sum_{i=1}^{N} \sum_{j \neq i}^{N} \delta\left[x_{1}-X_{i}(t)\right] \delta\left[x_{2}-X_{j}(t)\right],
\end{gathered}
$$

etc. Here $X_{i}(t) \equiv\left\{\mathbf{R}_{i}(t), \mathbf{V}_{i}(t)\right\}$, while the $x_{i} \equiv\left\{\mathbf{r}_{i}, \mathbf{v}_{i}\right\}$ are field variable referring to the one-particle phase space. The average of $F_{1}\left(x_{1}, t\right)$ and $F_{2}\left(x_{1}, x_{2}, t\right)$ over the initial probability distribution of the system $\rho(\Gamma, 0)$, where $\Gamma \equiv\left\{X_{1}(t), \ldots, X_{N}(t)\right\}$, are the usual one-particle and two-particle distribution functions

$$
f_{1}\left(x_{1}, t\right)=\left\langle F_{1}\left(x_{1}, t\right)\right\rangle, \quad f_{2}\left(x_{1}, x_{2}, t\right)=\left\langle F_{2}\left(x_{1}, x_{2}, t\right)\right\rangle,
$$


where we have introduced the notation

$$
\langle G\rangle \equiv \int d \Gamma G(\Gamma) \rho(\Gamma, 0) .
$$

In the dilute limit, assuming molecular chaos, i.e. that no correlations exist between colliding particles, and that the sizes of the jumps due to the thermostat are small compared to the scale on which the distribution varies, the equation for the one-particle distribution function, $f_{1}\left(x_{1}, t\right)$, is the Boltzmann-Fokker-Planck equation [30]

$$
\left[\frac{\partial}{\partial t}+L^{(0)}\left(x_{1}\right)\right] f_{1}\left(x_{1}, t\right)=J\left[f_{1} \mid f_{1}\right]+\frac{\xi_{0}^{2}}{2} \frac{\partial^{2}}{\partial \mathbf{v}_{1}^{2}} f_{1}\left(x_{1}, t\right),
$$

where we have introduced the free streaming operator

$$
L^{(0)}\left(x_{1}\right)=\mathbf{v}_{1} \cdot \frac{\partial}{\partial \mathbf{r}_{1}} .
$$

The collisional term can be expressed as

$$
J\left[f_{1} \mid f_{1}\right]=\int d x_{2} \delta\left(\mathbf{r}_{12}\right) \bar{T}_{0}\left(\mathbf{v}_{1}, \mathbf{v}_{2}\right) f_{1}\left(x_{1}, t\right) f_{1}\left(x_{2}, t\right)
$$

in terms of the binary collision operator

$$
\bar{T}_{0}\left(\mathbf{v}_{1}, \mathbf{v}_{2}\right)=\sigma^{d-1} \int d \hat{\boldsymbol{\sigma}} \Theta\left(\hat{\boldsymbol{\sigma}} \cdot \mathbf{v}_{12}\right)\left(\hat{\boldsymbol{\sigma}} \cdot \mathbf{v}_{12}\right)\left[\alpha^{-2} b_{\hat{\boldsymbol{\sigma}}}^{-1}-1\right],
$$

where we have neglected the separation between the centers of colliding particles. The operator $b_{\hat{\sigma}}^{-1}$ changes the velocities to its right into the pre-collisional velocities

$$
\begin{aligned}
& \mathbf{v}_{1}^{*}=\mathbf{v}_{1}-\frac{1+\alpha}{2 \alpha}\left(\hat{\boldsymbol{\sigma}} \cdot \mathbf{v}_{12}\right) \hat{\boldsymbol{\sigma}}, \\
& \mathbf{v}_{2}^{*}=\mathbf{v}_{2}+\frac{1+\alpha}{2 \alpha}\left(\hat{\boldsymbol{\sigma}} \cdot \mathbf{v}_{12}\right) \hat{\boldsymbol{\sigma}} .
\end{aligned}
$$

The last term in Eq. (2.7), which does not appear in the free cooling case, is the typical diffusive Fokker-Planck contribution from the heating.

The two-particle distribution function is defined in such a way, see Eq. (2.4) and Eq. (2.5), that the quantity $f_{2}\left(x_{1}, x_{2}, t\right) d x_{1} d x_{2}$ is just the average number of pairs of particles in which one lies inside the differential volume $d x_{1}$ centered in $x_{1}$ and likewise, with $d x_{2}$ and $x_{2}$ for the second particle. This definition is easily generalized to higher $n$-particle distribution functions, $f_{n}\left(x_{1}, \ldots, x_{n}\right)$. The evolution equation for $f_{2}$, in the same limit in which the Boltzmann equation is valid, is [7]

$$
\begin{aligned}
& {\left[\frac{\partial}{\partial t}+L^{(0)}\left(x_{1}\right)+L^{(0)}\left(x_{2}\right)\right] f_{2}\left(x_{1}, x_{2}, t\right)=\delta\left(\mathbf{r}_{12}\right) \sigma^{d-1} \bar{T}_{0}\left(\mathbf{v}_{1}, \mathbf{v}_{2}\right) f_{2}\left(x_{1}, x_{2}, t\right)} \\
& +\sigma^{d-1} \int d x_{3}\left[\delta\left(\mathbf{r}_{13}\right) \bar{T}_{0}\left(\mathbf{v}_{1}, \mathbf{v}_{3}\right)+\delta\left(\mathbf{r}_{23}\right) \bar{T}_{0}\left(\mathbf{v}_{2}, \mathbf{v}_{3}\right)\right] f_{3}\left(x_{1}, x_{2}, x_{3}, t\right)+F_{\mathrm{TH}}
\end{aligned}
$$


where we have introduced $F_{\mathrm{TH}}$ that accounts for the external driving. The evolution equation (2.13) contains essentially three parts: the free streaming in the left-hand side, the two terms in the right hand side corresponding to collisions, and the last term, $F_{\mathrm{TH}}$ due to the thermostat. The collisional contribution is split into one part corresponding to collisions of particles with velocities $\mathbf{v}_{1}$ and $\mathbf{v}_{2}$, and the other which involves collisions of particles with velocities $\mathbf{v}_{1}$ or $\mathbf{v}_{2}$ with a third particle with arbitrary velocity, $\mathbf{v}_{3}$. The collisional contribution is identical to the one that appears in the absence of forcing [7]. We concentrate now on the new term, $F_{\mathrm{TH}}$. Assuming that the sizes of the jumps due to the thermostat are small compared to the scale in which the distribution $f_{2}$ varies, we can expand $F_{\mathrm{TH}}$ in the spirit of the Fokker-Planck description [29]

$$
\begin{aligned}
F_{\mathrm{TH}} & \simeq \frac{1}{2} \sum_{\beta, \gamma=1}^{d} \sum_{i, j=1}^{2} B_{i j, \beta \gamma} \frac{\partial}{\partial v_{i, \beta}} \frac{\partial}{\partial v_{j, \gamma}} f_{2}\left(x_{1}, x_{2}, t\right) \\
& =\frac{1}{2} \xi_{0}^{2}\left[\frac{\partial^{2}}{\partial v_{1}^{2}}+\frac{\partial^{2}}{\partial v_{2}^{2}}-\frac{2}{N} \frac{\partial}{\partial \mathbf{v}_{1}} \cdot \frac{\partial}{\partial \mathbf{v}_{2}}\right] f_{2}\left(x_{1}, x_{2}, t\right)
\end{aligned}
$$

where we have taken into account equation (2.2), and we have explicitly assumed that the jump moments $B_{i j, \beta \gamma}$ do not depend on the magnitude of the velocities of the particles.

Two-time reduced distribution functions can also be defined in terms of the microscopic densities as

$$
f_{r, s}\left(x_{1}, \ldots, x_{r}, t ; x_{1}^{\prime}, \ldots, x_{s}^{\prime}, t^{\prime}\right) \equiv\left\langle F_{r}\left(x_{1}, \ldots, x_{r}, t\right) F_{s}\left(x_{1}^{\prime}, \ldots, x_{s}^{\prime}, t^{\prime}\right)\right\rangle,
$$

where it will be assumed that $t>t^{\prime}>0$, for concreteness. Again, in the low-density limit and taking into account the influence of the thermostat, the lowest order distribution, $f_{1,1}$, is seen to obey the equation [7],

$$
\begin{aligned}
{\left[\frac{\partial}{\partial t}+L^{(0)}\left(x_{1}\right)\right] f_{1,1}\left(x_{1}, t ; x_{1}^{\prime}, t^{\prime}\right) } & =\sigma^{d-1} \int d x_{2} \delta\left(\mathbf{r}_{12}\right) \bar{T}_{0}\left(\mathbf{v}_{1}, \mathbf{v}_{2}\right) f_{2,1}\left(x_{1}, x_{2}, t ; x_{1}^{\prime}, t^{\prime}\right) \\
& +\frac{\xi_{0}^{2}}{2} \frac{\partial^{2}}{\partial \mathbf{v}_{1}^{2}} f_{1,1}\left(x_{1}, t ; x_{1}^{\prime}, t^{\prime}\right)
\end{aligned}
$$

which has to be solved with the initial condition

$$
f_{1,1}\left(x_{1}, t^{\prime} ; x_{1}^{\prime}, t^{\prime}\right)=\delta\left(x_{1}-x_{1}^{\prime}\right) f_{1}\left(x_{1}, t^{\prime}\right)+f_{2}\left(x_{1}, x_{1}^{\prime}, t^{\prime}\right) .
$$

Let us introduce the two-particle and three-particle correlation functions, $g_{2}$ and $g_{3}$, through the usual cluster expansion

$$
f_{2}\left(x_{1}, x_{2}, t\right)=f_{1}\left(x_{1}, t\right) f_{1}\left(x_{2}, t\right)+g_{2}\left(x_{1}, x_{2}, t\right),
$$

and

$$
\begin{array}{r}
f_{3}\left(x_{1}, x_{2}, x_{3}, t\right)=f_{1}\left(x_{1}, t\right) f_{1}\left(x_{2}, t\right) f_{1}\left(x_{3}, t\right)+g_{2}\left(x_{1}, x_{2}, t\right) f_{1}\left(x_{3}, t\right) \\
\quad+g_{2}\left(x_{1}, x_{3}, t\right) f_{1}\left(x_{2}, t\right)+g_{2}\left(x_{2}, x_{3}, t\right) f_{1}\left(x_{1}, t\right)+g_{3}\left(x_{1}, x_{2}, x_{3}, t\right) .
\end{array}
$$


Similarly, for the two-time correlation functions, we introduce the two-time correlation functions $h_{1,1}$ and $h_{2,1}$ through

$$
\begin{aligned}
f_{1,1}\left(x_{1}, t ;\right. & \left.x_{1}^{\prime}, t^{\prime}\right)=f_{1}\left(x_{1}, t\right) f_{1}\left(x_{1}^{\prime}, t^{\prime}\right)+h_{1,1}\left(x_{1}, t ; x_{1}^{\prime}, t^{\prime}\right) \\
f_{2,1}\left(x_{1}, x_{2}, t ; x_{1}^{\prime}, t^{\prime}\right) & =f_{1}\left(x_{1}, t\right) f_{1}\left(x_{2}, t\right) f_{1}\left(x_{1}^{\prime}, t^{\prime}\right)+g_{2}\left(x_{1}, x_{2}, t\right) f_{1}\left(x_{1}^{\prime}, t^{\prime}\right) \\
& +h_{1,1}\left(x_{1}, t ; x_{1}^{\prime}, t^{\prime}\right) f_{1}\left(x_{2}, t\right)+h_{1,1}\left(x_{2}, t ; x_{1}^{\prime}, t^{\prime}\right) f_{1}\left(x_{1}, t\right) \\
& +h_{2,1}\left(x_{1}, x_{2}, t ; x_{1}^{\prime}, t^{\prime}\right) .
\end{aligned}
$$

The equation for the correlation function $g_{2}\left(x_{1}, x_{2}, t\right)$ can be obtained following the same lines as in reference [7]. Taking into account Eq. (2.18) and neglecting the three-body correlations, $g_{3}$, in Eq. (2.13), we obtain

$$
\begin{array}{r}
{\left[\frac{\partial}{\partial t}+L^{(0)}\left(x_{1}\right)+L^{(0)}\left(x_{2}\right)\right] g_{2}\left(x_{1}, x_{2}, t\right)=\delta\left(\mathbf{r}_{12}\right) \sigma^{d-1} \bar{T}_{0}\left(\mathbf{v}_{1}, \mathbf{v}_{2}\right) f_{1}\left(x_{1}, t\right) f_{1}\left(x_{2}, t\right)} \\
+\left[K\left(x_{1}, t\right)+K\left(x_{2}, t\right)\right] g_{2}\left(x_{1}, x_{2}, t\right)-\frac{\xi_{0}^{2}}{N} \frac{\partial}{\partial \mathbf{v}_{1}} \cdot \frac{\partial}{\partial \mathbf{v}_{2}} f_{1}\left(x_{1}, t\right) f_{1}\left(x_{2}, t\right),
\end{array}
$$

where we have introduced the linear operator $K\left(x_{i}, t\right)$ defined as

$$
K\left(x_{i}, t\right)=\sigma^{d-1} \int d x_{3} \delta\left(\mathbf{r}_{i 3}\right) \bar{T}_{0}\left(\mathbf{v}_{i}, \mathbf{v}_{3}\right)\left(1+\mathcal{P}_{i 3}\right) f_{1}\left(x_{3}, t\right)+\frac{\xi_{0}^{2}}{2}\left(\frac{\partial}{\partial \mathbf{v}_{i}}\right)^{2},
$$

and where the permutation operator $\mathcal{P}_{a b}$ interchanges the labels of particles $a$ and $b$ in the quantities on which it acts.

In the same way, the equation for $h_{1,1}$, neglecting the contribution coming from $h_{2,1}$ in Eq. (2.16), is

$$
\left[\frac{\partial}{\partial t}+L^{(0)}\left(x_{1}\right)-K\left(x_{1}, t\right)\right] h_{1,1}\left(x_{1}, t ; x_{1}^{\prime}, t^{\prime}\right)=0
$$

which have to be solved with the initial condition

$$
h_{1,1}\left(x_{1}, t ; x_{1}^{\prime}, t^{\prime}\right)=\delta\left(x_{1}-x_{1}^{\prime}\right) f_{1}\left(x_{1}, t^{\prime}\right)+g_{2}\left(x_{1}, x_{1}^{\prime}, t^{\prime}\right) .
$$

To sum up, under the same hypothesis made to derive the Boltzmann equation, we obtain closed equations for the one-time and two-time correlation functions, Eqs. (2.22) and (2.24). These equations represent a starting point for the study of the fluctuations and correlations in the heated system.

\subsection{The stationary state}

It has been shown numerically that, for a wide class of initial conditions, the system reaches a homogeneous stationary state [31] in which the energy input from the thermostat is compensated by the energy lost in collisions. In this case the Boltzmann-Fokker-Planck equation reads

$$
\frac{\xi_{0}^{2}}{2} \frac{\partial^{2}}{\partial \mathbf{v}_{1}^{2}} f_{H}\left(\mathbf{v}_{1}\right)+J\left[f_{H} \mid f_{H}\right]=0
$$


For the sake of simplicity, let us introduce the following dimensionless distribution

$$
f_{H}(\mathbf{v})=\frac{n_{H}}{v_{H}^{d}} \chi_{H}(c),
$$

with $\chi_{H}(c)$ an isotropic function of the modulus of $\mathbf{c}, v_{H}=\left(\frac{2 T_{H}}{m}\right)^{1 / 2}, \mathbf{c}=\frac{\mathbf{v}}{v_{H}}$ and $T_{H}$ the granular temperature defined as

$$
\frac{d}{2} n_{H} T_{H}=\int d \mathbf{v} \frac{1}{2} m v^{2} f_{H}(\mathbf{v})
$$

By introducing equation (2.27) in equation (2.26), we obtain a closed equation for $\chi_{H}$

$$
\int d \mathbf{c}_{2} \bar{T}\left(\mathbf{c}_{1}, \mathbf{c}_{2}\right) \chi_{H}\left(\mathbf{c}_{1}\right) \chi_{H}\left(\mathbf{c}_{2}\right)+\frac{\xi^{2}}{2} \frac{\partial}{\partial \mathbf{c}_{1}^{2}} \chi_{H}\left(c_{1}\right)=0
$$

where

$$
\bar{T}\left(\mathbf{c}_{1}, \mathbf{c}_{2}\right)=\int d \hat{\boldsymbol{\sigma}} \Theta\left(\hat{\boldsymbol{\sigma}} \cdot \mathbf{c}_{12}\right)\left(\hat{\boldsymbol{\sigma}} \cdot \mathbf{c}_{12}\right)\left[\alpha^{-2} b_{\hat{\boldsymbol{\sigma}}}^{-1}-1\right]
$$

is the dimensionless binary collision operator and $\xi^{2}=\frac{\xi_{0}^{2} \ell}{v_{H}^{3}}$ is the dimensionless strength of the noise with $\ell=\left(n_{H} \sigma^{d-1}\right)^{-1}$ proportional to the mean free path.

An approximate expression for the scaled one-particle distribution function $\chi_{H}(c)$ was derived to second order in Sonine polynomials [30]

$$
\chi_{H}(c)=\frac{e^{-c^{2}}}{\pi^{d / 2}}\left[1+a_{2}(\alpha) S_{d / 2-1}^{2}\left(c^{2}\right)\right]
$$

where

$$
S_{d / 2-1}^{2}\left(c^{2}\right)=\frac{1}{2} c^{4}-\frac{1}{2}(d+2) c^{2}+\frac{1}{8} d(d+2) .
$$

The coefficient $a_{2}(\alpha)$ is related to the forth velocity moment of $\chi_{H}(c)$

$$
\frac{d}{d+2} \frac{\left\langle c^{4}\right\rangle_{H}}{\left\langle c^{2}\right\rangle_{H}^{2}}=1+a_{2}(\alpha)
$$

and an approximate expression is [30]

$$
a_{2}=\frac{16(1-\alpha)\left(1-2 \alpha^{2}\right)}{73+56 d-24 \alpha d-105 \alpha+30(1-\alpha) \alpha^{2}} .
$$

In the stationary state, the expression for the temperature in the first Sonine approximation is given by

$$
T_{H}=m\left[\frac{d \xi_{0}^{2} \sqrt{\pi}}{\left(1-\alpha^{2}\right) \Omega_{d} n_{H} \sigma^{d-1}}\right]^{2 / 3}\left(1+\mathcal{O}\left(a_{2}\right)\right),
$$

where $\Omega_{d}=2 \pi^{d / 2} / \Gamma(d / 2)$ is the $d$-dimensional solid angle. 
In the case of the correlation functions for this homogeneous state, $g_{2}^{H}$ and $h_{1,1}^{H}$, it is convenient to introduce the dimensionless counterparts, $g_{H}$ and $h_{H}$, as

$$
g_{2}^{H}\left(x_{1}, x_{2}\right)=\frac{n_{H}}{\ell^{d} v_{H}^{2 d}} g_{H}\left(\mathbf{l}_{12}, \mathbf{c}_{1}, \mathbf{c}_{2}\right), \quad h_{1,1}^{H}\left(x_{1}, t ; x_{2}, t^{\prime}\right)=\frac{n_{H}}{\ell^{d} v_{H}^{2 d}} h_{H}\left(\mathbf{l}_{12}, \mathbf{c}_{1}, \mathbf{c}_{2}, s-s^{\prime}\right),
$$

where we have introduced the dimensionless length scale $\mathbf{l}=\mathbf{r} / \ell, \mathbf{l}_{12}=\mathbf{l}_{1}-\mathbf{l}_{2}$ and the dimensionless time-scale $s=\frac{v_{H}}{\ell} t$. The reduced distribution, $g_{H}$, fulfills

$$
\begin{aligned}
{\left[\Lambda\left(\mathbf{c}_{1}\right)+\Lambda\left(\mathbf{c}_{2}\right)-\mathbf{c}_{12} \cdot \frac{\partial}{\partial \mathbf{l}_{12}}\right] g_{H}\left(\mathbf{l}_{12}, \mathbf{c}_{1}, \mathbf{c}_{2}\right) } & =-\delta\left(\mathbf{l}_{12}\right) \bar{T}\left(\mathbf{c}_{1}, \mathbf{c}_{2}\right) \chi_{H}\left(c_{1}\right) \chi_{H}\left(c_{2}\right) \\
& +\xi^{2} \frac{n_{H} \ell^{d}}{N} \frac{\partial}{\partial \mathbf{c}_{1}} \cdot \frac{\partial}{\partial \mathbf{c}_{2}} \chi_{H}\left(c_{1}\right) \chi_{H}\left(c_{2}\right)
\end{aligned}
$$

where $\Lambda\left(\mathbf{c}_{i}\right)$ is the linearized Boltzmann-Fokker-Planck operator

$$
\Lambda\left(\mathbf{c}_{i}\right) h\left(\mathbf{c}_{i}\right)=\int d \mathbf{c}_{3} \bar{T}\left(\mathbf{c}_{i}, \mathbf{c}_{3}\right)\left(1+\mathcal{P}_{i 3}\right) \chi_{H}\left(c_{3}\right) h\left(\mathbf{c}_{i}\right)+\frac{\xi^{2}}{2} \frac{\partial^{2}}{\partial \mathbf{c}_{i}^{2}} h\left(\mathbf{c}_{i}\right) .
$$

Equation (2.37) describes the one-time correlation between fluctuations in the stationary state. As can be seen, the correlation function is determined by the properties of the linearized BoltzmannFokker-Planck operator, $\Lambda$, and by the one-particle distribution function, $\chi_{H}$.

The evolution equation for the two-time correlation function is

$$
\left[\frac{\partial}{\partial s}-\Lambda\left(\mathbf{c}_{1}\right)+\mathbf{c}_{1} \cdot \frac{\partial}{\partial \mathbf{l}_{1}}\right] h_{H}\left(\mathbf{l}_{12}, \mathbf{c}_{1}, \mathbf{c}_{2}, s-s^{\prime}\right)=0
$$

which has to be solved with the initial condition

$$
h_{H}\left(\mathbf{l}_{12}, \mathbf{c}_{1}, \mathbf{c}_{2}, 0\right) \equiv h_{H}\left(\mathbf{l}_{12}, \mathbf{c}_{1}, \mathbf{c}_{2}\right)=\chi_{H}\left(c_{1}\right) \delta\left(\mathbf{l}_{12}\right) \delta\left(\mathbf{c}_{12}\right)+g_{H}\left(\mathbf{l}_{12}, \mathbf{c}_{1}, \mathbf{c}_{2}\right) .
$$

Taking into account equation (2.37) and that the term $\mathbf{c}_{12} \cdot \frac{\partial}{\partial \mathbf{l}_{12}} \chi_{H}\left(c_{1}\right) \delta\left(\mathbf{l}_{12}\right) \delta\left(\mathbf{c}_{12}\right)$ vanishes identically, the equation for this quantity is

$$
\begin{aligned}
{\left[\Lambda\left(\mathbf{c}_{1}\right)+\right.} & \left.\Lambda\left(\mathbf{c}_{2}\right)-\mathbf{c}_{12} \cdot \frac{\partial}{\partial \mathbf{l}_{12}}\right] h_{H}\left(\mathbf{l}_{12}, \mathbf{c}_{1}, \mathbf{c}_{2}\right) \\
& =-\delta\left(\mathbf{l}_{12}\right) \Gamma\left(\mathbf{c}_{1}, \mathbf{c}_{2}\right)+\xi^{2} \frac{n_{H} \ell^{d}}{N} \frac{\partial}{\partial \mathbf{c}_{1}} \cdot \frac{\partial}{\partial \mathbf{c}_{2}} \chi_{H}\left(c_{1}\right) \chi_{H}\left(c_{2}\right),
\end{aligned}
$$

where

$$
\Gamma\left(\mathbf{c}_{1}, \mathbf{c}_{2}\right)=\bar{T}\left(\mathbf{c}_{1}, \mathbf{c}_{2}\right) \chi_{H}\left(c_{1}\right) \chi_{H}\left(c_{2}\right)-\left[\Lambda\left(\mathbf{c}_{1}\right)+\Lambda\left(\mathbf{c}_{2}\right)\right] \chi_{H}\left(c_{1}\right) \delta\left(\mathbf{c}_{12}\right)
$$




\section{Hydrodynamics}

Let us summarize briefly how the system can be analyzed from a hydrodynamic point of view. The hydrodynamic fields can be defined as usually done in kinetic theory

$$
\begin{aligned}
n(\mathbf{r}, t) & =\int d \mathbf{v} f_{1}(x, t), \\
n(\mathbf{r}, t) \mathbf{u}(\mathbf{r}, t) & =\int d \mathbf{v} \mathbf{v} f_{1}(x, t), \\
\frac{d}{2} n(\mathbf{r}, t) T(\mathbf{r}, t) & =\int d \mathbf{v} \frac{m}{2} \mathbf{V}(\mathbf{r}, t)^{2} f_{1}(x, t),
\end{aligned}
$$

where the function $\mathbf{V}(\mathbf{r}, t) \equiv \mathbf{v}-\mathbf{u}(\mathbf{r}, t)$ is the peculiar velocity. The complete non-linear equations for these fields for a granular system heated by the stochastic thermostat are $[15,31]$

$$
\begin{aligned}
\frac{\partial}{\partial t} n & =-\nabla \cdot(n \mathbf{u}) \\
\frac{\partial}{\partial t} \mathbf{u} & =-\mathbf{u} \cdot \nabla \mathbf{u}-\frac{1}{m n} \nabla_{j} P_{i j}, \\
\frac{\partial}{\partial t} T & =-\mathbf{u} \cdot \nabla T-\frac{2}{d n}\left(\nabla \cdot \mathbf{q}+P_{i j} \nabla_{j} u_{i}\right)-\zeta T+m \xi_{0}^{2}
\end{aligned}
$$

where $P_{i j}$ is the pressure tensor, $\mathbf{q}$ is the heat flux and $\zeta$ is the cooling rate, which is also a functional of the distribution function

$$
\zeta=\frac{\left(1-\alpha^{2}\right) m \pi^{\frac{d-1}{2}} \sigma^{d-1}}{4 d \Gamma\left(\frac{d+3}{2}\right) n k_{B} T} \int d \mathbf{v}_{1} \int d \mathbf{v}_{2}\left|\mathbf{v}_{1}-\mathbf{v}_{2}\right|^{3} f_{1}\left(\mathbf{r}, \mathbf{v}_{1}, t\right) f_{1}\left(\mathbf{r}, \mathbf{v}_{2}, t\right) .
$$

\subsection{Evolution of homogeneous perturbations}

Now, we will focus on the study of a linear perturbation of the hydrodynamic fields around the homogeneous stationary state. We are interested, primarily, in the information related to the hydrodynamic eigenvalues that we can obtain from them.

Considering a homogeneous state, the previous equations reduce to

$$
\frac{\partial}{\partial t} n=0, \quad \frac{\partial}{\partial t} \mathbf{u}=\mathbf{0}, \quad \frac{\partial}{\partial t} T=-\zeta T+m \xi_{0}^{2} .
$$

In the long time limit, the system is expected to approach a steady state with a constant temperature given by the equation

$$
\zeta_{H}\left(f_{H}\right) T_{H}=m \xi_{0}^{2}
$$

Substituting the explicit form of the scaled one-particle distribution function in the first Sonine approximation, Eq. (2.31), in the equation above, we obtain the temperature given in (2.35). 
Let us consider now a homogeneous state close to this homogeneous stationary state. We can write the hydrodynamic fields as $n(t)=n_{H}+\delta n, \mathbf{u}(t)=\delta \mathbf{u}$ and $T(t)=T_{H}+\delta T$. We also define the dimensionless hydrodynamic fields

$$
\delta \rho(s)=\frac{\delta n}{n_{H}}, \quad \delta \mathbf{w}(s)=\frac{\delta \mathbf{u}}{v_{H}}, \quad \delta \theta(s)=\frac{\delta T}{T_{H}},
$$

where we used the dimensionless time scale $s$ introduced in the previous section. Assuming that the deviations are small, and taking into account equations (3.8)-(3.9), we can write the linearized evolution equations for the dimensionless hydrodynamic fields in this new time scale

$$
\frac{\partial}{\partial s} \delta \rho=0, \quad \frac{\partial}{\partial s} \delta \mathbf{w}=0, \quad \frac{\partial}{\partial s} \delta \theta=-\zeta_{0} \delta \rho-\frac{3}{2} \zeta_{0} \delta \theta
$$

where $\zeta_{0}=\frac{l \zeta_{H}}{v_{H}}$ is a dimensionless coefficient that is a functional of the one-particle distribution function in the stationary state. Its expression in the first Sonine approximation is [30]

$$
\zeta_{0}=\frac{\left(16+3 a_{2}\right) \pi^{\frac{d-1}{2}}\left(1-\alpha^{2}\right)}{8 \sqrt{2} d \Gamma\left(\frac{d}{2}\right)} .
$$

To obtain the equation for $\delta \theta$ we have assumed that the perturbed distribution function scales as

$$
f_{1}(\mathbf{v}, t)=\frac{n}{\bar{v}(t)^{d}} \chi_{H}\left(\frac{\mathbf{v}}{\bar{v}(t)}\right)
$$

where $\bar{v}(t)=\left[\frac{2 T(t)}{m}\right]^{1 / 2}$, and $\chi_{H}$ is the same scaled distribution function as for the reference stationary state. This assumption has already been used and tested numerically in [31], but we remark that it is an additional approximation. Then, the cooling rate $\zeta$ for the state under scrutiny is proportional to $T^{1 / 2}(t)$ and we obtain the equation for the linearized energy written above. Equations (3.11) indicate that a perturbation in the total number of particles or total momentum does not decay, as a consequence of the fact that these variables are conserved, but a perturbation in the total energy will decay exponentially to the stationary value, as expected. Moreover, as the equation for the temperature can be rewritten in the following form

$$
\frac{\partial}{\partial s}\left(\frac{2}{3} \delta \rho+\delta \theta\right)=-\frac{3}{2} \zeta_{0}\left(\frac{2}{3} \delta \rho+\delta \theta\right)
$$

we can identify the hydrodynamic eigenvalues $\lambda=0$ and $\gamma=-\frac{3}{2} \zeta_{0}, \lambda$ being $(d+1)$-fold degenerate. For the sake of clarity, it proves convenient to relabel these eigenvalues as

$$
\lambda_{1}=0, \quad \lambda_{2}=0, \quad \lambda_{3}=-\frac{3}{2} \zeta_{0},
$$

where $\lambda_{2}$ is $d$-fold degenerate. The associated hydrodynamic modes, $\left\{y_{\beta}\right\}_{\beta=1}^{d+2}$ are

$$
y_{1}=\delta \rho, \quad \mathbf{y}_{2}=\delta \mathbf{w}, \quad y_{3}=\frac{2}{3} \delta \rho+\delta \theta .
$$




\subsection{Enforcing consistency with the linearized Boltzmann-Fokker-Planck equation description}

We now turn our attention to the problem of finding the linearized hydrodynamic equations for a homogeneous perturbation, directly from the Boltzmann-Fokker-Planck equation. Enforcing consistency with the macroscopic considerations of section 3.1. above, we will infer useful properties on the hydrodynamic part of the spectrum of $\Lambda(\mathbf{c})$. We first introduce the scaled deviation of the distribution function

$$
\delta \chi(\mathbf{c}, s)=\frac{v_{H}^{d}}{n_{H}}\left[f_{1}(\mathbf{v}, t)-f_{H}(\mathbf{v})\right] .
$$

The evolution of the scaled distribution is governed by

$$
\frac{\partial}{\partial s} \delta \chi(\mathbf{c}, s)=\Lambda(\mathbf{c}) \delta \chi(\mathbf{c}, s)
$$

where the operator $\Lambda(\mathbf{c})$ is the linearized Boltzmann-Fokker-Planck operator defined in (2.38). Let us also introduce the scalar product

$$
\langle f(\mathbf{c}) \mid g(\mathbf{c})\rangle \equiv \int d \mathbf{c} \chi_{H}^{-1}(c) f^{*}(\mathbf{c}) g(\mathbf{c}),
$$

where $f^{*}$ is the complex conjugate of $f$. Interestingly, the hydrodynamic modes introduced in (3.16) can then be written as

$$
y_{\beta}=\left\langle\bar{\xi}_{\beta} \mid \delta \chi\right\rangle, \quad \beta=1,2,3,
$$

where

$$
\bar{\xi}_{1}(\mathbf{c})=\chi_{H}(c), \quad \overline{\boldsymbol{\xi}}_{2}(\mathbf{c})=\mathbf{c} \chi_{H}(c), \quad \bar{\xi}_{3}(\mathbf{c})=\left(\frac{c^{2}}{d}-\frac{1}{6}\right) \chi_{H}(c) .
$$

Taking the scalar product of the linearized Boltzmann-Fokker-Planck equation (3.18) with the functions $\bar{\xi}_{\beta}$, we obtain the linear equations (3.11) (in the hydrodynamic time scale, that is, if we wait long enough so that fast modes have vanished) only if the spectrum of $\Lambda$ admits the three eigenvalues written in (3.15), and the associated "hydrodynamic" eigenfunctions, $\left\{\xi_{\beta}\right\}_{\beta=1}^{d+2}$, obey the orthogonality condition

$$
\left\langle\bar{\xi}_{\beta_{1}} \mid \xi_{\beta_{2}}\right\rangle=\delta_{\beta_{1} \beta_{2}}, \quad \beta_{1}, \beta_{2}=1,2,3
$$

In Appendix A, it is shown that the null eigenvalue is $(d+1)$-fold degenerate, and the corresponding eigenfunctions, $\xi_{1}$ and $\boldsymbol{\xi}_{2}$, are worked out. Moreover, as a consequence of particle and total momentum conservation in a collision, $\bar{\xi}_{1}$ and $\overline{\boldsymbol{\xi}}_{2}$ are the corresponding left eigenfunctions. We were not able to demonstrate that $\lambda_{3}$ is an eigenvalue of $\Lambda$, but we have shown explicitly that

$$
\left\langle\bar{\xi}_{3} \mid \xi_{\beta}\right\rangle=0, \quad \text { for } \quad \beta=1,2 \text {. }
$$

In the following, we will assume that $\Lambda$ actually admits this third eigenvalue, with an unknown eigenfunction $\xi_{3}$ and that $\bar{\xi}_{3}$ is a good approximation of the actual left eigenfunction associated 
with $\lambda_{3}$. With the help of this assumption, we will see in the following sections that it is possible to define a projector in the hydrodynamic subspace. Let us remark that for inelastic Maxwell particles in the free cooling case the function $\bar{\xi}_{3}$ identified in this way is the actual left eigenfunction [4]. For the heated case the situation is similar and the linearized Boltzmann-Fokker-Planck operator for inelastic Maxwell particles has a left eigenfunction which is a linear combination of the two functions $\chi_{H}(c)$, and $c^{2} \chi_{H}(c)$ (in fact, the linear combination which makes the function orthogonal to $\left.\xi_{1}(\mathbf{c})\right)$. These facts support our assumption as a reasonable approximation for the hard particle case.

\section{The Boltzmann-Langevin equation}

The starting point for the study of the fluctuations in this work will be the Boltzmann-Langevin equation. In this section we derive the corresponding equation and we determine the properties of the noise in order to obtain consistency with the equations of the correlation functions presented in section 2 ..

As defined in Eq. (2.5), the one-particle distribution function, $f_{1}$, is the ensemble average of the phase function $F_{1}$, and its dynamics is given by the Boltzmann-Fokker-Planck equation, Eq. (2.7). The problem is now to find an evolution equation for the fluctuating quantity

$$
\delta F(\mathbf{l}, \mathbf{c}, s)=\frac{v_{H}^{d}}{n_{H}}\left[F_{1}(x, t)-f_{H}(\mathbf{v})\right] .
$$

As for the velocity of a Brownian particle [28], we expect that the difference between the equation for the fluctuating quantity $\delta F$, and its average, $\langle\delta F\rangle=\delta \chi$, is a random force term, $R$ [2]. Then, the fluctuations $\delta F$ around $\chi_{H}$ are described by a Boltzmann equation linearized around the solution $\chi_{H}$ with a random force, $R$, added

$$
\frac{\partial}{\partial s} \delta F(\mathbf{l}, \mathbf{c}, s)=\left[\Lambda(\mathbf{c})-\mathbf{c} \cdot \frac{\partial}{\partial \mathbf{l}}\right] \delta F(\mathbf{l}, \mathbf{c}, s)+R(\mathbf{l}, \mathbf{c}, s) .
$$

Taking averages in equation (4.2), we obtain the linearized Boltzmann-Fokker-Planck equation if and only if

$$
\langle R(\mathbf{l}, \mathbf{c}, s)\rangle=0
$$

Equation (4.2) is the Boltzmann-Langevin equation. As in the free cooling case [6], we assume that the noise term, $R(\mathbf{l}, \mathbf{c}, s)$, is Markovian

$$
\left\langle R\left(\mathbf{l}_{1}, \mathbf{c}_{1}, s_{1}\right) R\left(\mathbf{l}_{2}, \mathbf{c}_{2}, s_{2}\right)\right\rangle_{H}=H\left(\mathbf{l}_{1}, \mathbf{l}_{2}, \mathbf{c}_{1}, \mathbf{c}_{2}\right) \delta\left(s_{1}-s_{2}\right),
$$

where $\langle\ldots\rangle_{H}$ means average in the stationary homogeneous state. In order to evaluate the function $H\left(\mathbf{l}_{1}, \mathbf{l}_{2}, \mathbf{c}_{1}, \mathbf{c}_{2}\right)$ explicitly, we will calculate $\left\langle\delta F\left(\mathbf{l}_{1}, \mathbf{c}_{1}, s\right) \delta F\left(\mathbf{l}_{2}, \mathbf{c}_{2}, s\right)\right\rangle_{H}$ with the BoltzmannLangevin equation and then, we will impose compatibility with the equation of the correlation function of Sec. 2.. So, let us first write this function as a functional of the distribution and the 
correlation functions. Using the definitions of the microscopic densities, Eqs. (2.3), (2.4), the distribution and correlation functions, Eqs. (2.5), (2.18), (2.40), and the dimensionless distributions, Eqs. (2.27), (2.36), we have

$$
\begin{aligned}
\left\langle\delta F\left(\mathbf{l}_{1}, \mathbf{c}_{1}, s\right) \delta F\left(\mathbf{l}_{2}, \mathbf{c}_{2}, s\right)\right\rangle_{H} & =\frac{v_{H}^{2 d}}{n_{H}^{2}}\left\langle\left[F_{1}\left(x_{1}, t\right)-f_{H}\left(\mathbf{v}_{1}\right)\right]\left[F_{1}\left(x_{2}, t\right)-f_{H}\left(\mathbf{v}_{2}\right)\right]\right\rangle_{H} \\
& =\frac{v_{H}^{2 d}}{n_{H}^{2}}\left[\left\langle F_{1}\left(x_{1}, t\right) F_{1}\left(x_{2}, t\right)\right\rangle_{H}-f_{H}\left(\mathbf{v}_{1}\right) f_{H}\left(\mathbf{v}_{2}\right)\right] \\
& =\frac{v_{H}^{2 d}}{n_{H}^{2}}\left[f_{2, H}\left(x_{1}, x_{2}, t\right)+f_{H}\left(\mathbf{v}_{1}\right) \delta\left(x_{1}-x_{2}\right)-f_{H}\left(\mathbf{v}_{1}\right) f_{H}\left(\mathbf{v}_{2}\right)\right] \\
& =\frac{v_{H}^{2 d}}{n_{H}^{2}}\left[g_{2, H}\left(x_{1}, x_{2}\right)+f_{H}\left(\mathbf{v}_{1}\right) \delta\left(x_{1}-x_{2}\right)\right] \\
& =\frac{1}{n_{H} \ell^{d}}\left[g_{H}\left(\mathbf{l}_{12}, \mathbf{c}_{1}, \mathbf{c}_{2}\right)+\delta\left(\mathbf{l}_{12}\right) \delta\left(\mathbf{c}_{12}\right) \chi_{H}\left(c_{1}\right)\right] \\
& =\frac{1}{n_{H} \ell^{d}} h_{H}\left(\mathbf{l}_{12}, \mathbf{c}_{1}, \mathbf{c}_{2}\right) .
\end{aligned}
$$

Now, let us solve Eq. (4.2) as a functional of the noise. In order to do that it is convenient to define the linear operator

$$
\Lambda\left(\mathbf{l}_{i}, \mathbf{c}_{i}\right) \equiv \Lambda\left(\mathbf{c}_{i}\right)-\mathbf{c}_{i} \cdot \frac{\partial}{\partial \mathbf{l}_{i}},
$$

in terms of which the solution for $\delta F(\mathbf{l}, \mathbf{c}, s)$ is

$$
\begin{aligned}
\delta F(\mathbf{l}, \mathbf{c}, s)= & e^{\Lambda(\mathbf{l}, \mathbf{c}) s} \delta F(\mathbf{l}, \mathbf{c}, 0)+\int_{0}^{s} d s^{\prime} e^{\Lambda(\mathbf{l}, \mathbf{c})\left(s-s^{\prime}\right)} R\left(\mathbf{l}, \mathbf{c}, s^{\prime}\right) \\
& \stackrel{s \gg 1}{\longrightarrow} \int_{0}^{s} d s^{\prime} e^{\Lambda(\mathbf{l}, \mathbf{c})\left(s-s^{\prime}\right)} R\left(\mathbf{l}, \mathbf{c}, s^{\prime}\right),
\end{aligned}
$$

where we have assumed that the term stemming from the initial conditions vanishes in the long time limit. This is equivalent to assuming that the spectrum of the linearized Boltzmann-FokkerPlanck operator is such that any perturbation without component in the subspace associated with the null eigenvalue decays. With equation (4.7), we can evaluate

$$
\begin{aligned}
&\left\langle\delta F\left(\mathbf{l}_{1}, \mathbf{c}_{1}, s\right) \delta F\left(\mathbf{l}_{2}, \mathbf{c}_{2}, s\right)\right\rangle_{H} \\
&= \int_{0}^{s} d s^{\prime} \int_{0}^{s} d s^{\prime \prime} e^{\Lambda\left(\mathbf{l}_{1}, \mathbf{c}_{1}\right)\left(s-s^{\prime}\right)+\Lambda\left(\mathbf{l}_{2}, \mathbf{c}_{2}\right)\left(s-s^{\prime \prime}\right)}\left\langle R\left(\mathbf{l}_{1}, \mathbf{c}_{1}, s^{\prime}\right) R\left(\mathbf{l}_{2}, \mathbf{c}_{2}, s^{\prime \prime}\right)\right\rangle_{H} \\
&= \int_{0}^{s} d s^{\prime} \int_{0}^{s} d s^{\prime \prime} e^{\Lambda\left(\mathbf{l}_{1}, \mathbf{c}_{1}\right)\left(s-s^{\prime}\right)+\Lambda\left(\mathbf{l}_{2}, \mathbf{c}_{2}\right)\left(s-s^{\prime \prime}\right)} H\left(\mathbf{l}_{12}, \mathbf{c}_{1}, \mathbf{c}_{2}\right) \delta\left(s^{\prime}-s^{\prime \prime}\right) \\
&= \int_{0}^{s} d s^{\prime} e^{\left[\Lambda\left(\mathbf{l}_{1}, \mathbf{c}_{1}\right)+\Lambda\left(\mathbf{l}_{2}, \mathbf{c}_{2}\right)\right]\left(s-s^{\prime}\right)} H\left(\mathbf{l}_{12}, \mathbf{c}_{1}, \mathbf{c}_{2}\right) \\
&=-\left[\Lambda\left(\mathbf{l}_{1}, \mathbf{c}_{1}\right)+\Lambda\left(\mathbf{l}_{2}, \mathbf{c}_{2}\right)\right]^{-1}\left[e^{\left[\Lambda\left(\mathbf{l}_{1}, \mathbf{c}_{1}\right)+\Lambda\left(\mathbf{l}_{2}, \mathbf{c}_{2}\right)\right]\left(s-s^{\prime}\right)}\right]_{s^{\prime}=0}^{s^{\prime}=s} H\left(\mathbf{l}_{12}, \mathbf{c}_{1}, \mathbf{c}_{2}\right) \\
& \stackrel{s \gg 1}{\longrightarrow}-\left[\Lambda\left(\mathbf{l}_{1}, \mathbf{c}_{1}\right)+\Lambda\left(\mathbf{l}_{2}, \mathbf{c}_{2}\right)\right]^{-1} H\left(\mathbf{l}_{12}, \mathbf{c}_{1}, \mathbf{c}_{2}\right),
\end{aligned}
$$


where we have assumed that the term $e^{\left[\Lambda\left(\mathbf{l}_{1}, \mathbf{c}_{1}\right)+\Lambda\left(\mathbf{l}_{2}, \mathbf{c}_{2}\right)\right] s} H\left(\mathbf{l}_{12}, \mathbf{c}_{1}, \mathbf{c}_{2}\right) \rightarrow 0$ in the long time limit. After identifying the function, we will see that this is, in fact, the case, because $H\left(\mathbf{l}_{12}, \mathbf{c}_{1}, \mathbf{c}_{2}\right)$ does not have components in the subspace associated with the null eigenvalue. Equivalently we have

$$
\left[\Lambda\left(\mathbf{l}_{1}, \mathbf{c}_{1}\right)+\Lambda\left(\mathbf{l}_{2}, \mathbf{c}_{2}\right)\right] h_{H}\left(\mathbf{l}_{12}, \mathbf{c}_{1}, \mathbf{c}_{2}\right)=-n_{H} \ell^{d} H\left(\mathbf{l}_{12}, \mathbf{c}_{1}, \mathbf{c}_{2}\right) .
$$

Finally, comparing equations (4.9) and (2.41) we conclude that

$$
H\left(\mathbf{l}_{12}, \mathbf{c}_{1}, \mathbf{c}_{2}\right)=\frac{1}{n_{H} \ell^{d}} \delta\left(\mathbf{l}_{12}\right) \Gamma\left(\mathbf{c}_{1}, \mathbf{c}_{2}\right)-\frac{\xi^{2}}{N} \frac{\partial}{\partial \mathbf{c}_{1}} \cdot \frac{\partial}{\partial \mathbf{c}_{2}} \chi_{H}\left(c_{1}\right) \chi_{H}\left(c_{2}\right),
$$

with $\Gamma\left(\mathbf{c}_{1}, \mathbf{c}_{2}\right)$ given in Eq. (2.42). With this expression of $H$ we can see that it does not have components in the subspace associated with the null eigenvalue. Taking into account that $\bar{\xi}_{1}$ and $\overline{\boldsymbol{\xi}}_{2}$ are left eigenfunctions of $\Lambda$ associated with the null eigenvalue and that [14]

$$
\begin{array}{r}
\int d \mathbf{c}_{1} \int d \mathbf{c}_{2} \bar{T}\left(\mathbf{c}_{1}, \mathbf{c}_{2}\right) \chi_{H}\left(\mathbf{c}_{1}\right) \chi_{H}\left(\mathbf{c}_{2}\right)=0 \\
\int d \mathbf{c}_{1} \int d \mathbf{c}_{2} c_{1 i} \bar{T}\left(\mathbf{c}_{1}, \mathbf{c}_{2}\right) \chi_{H}\left(\mathbf{c}_{1}\right) \chi_{H}\left(\mathbf{c}_{2}\right)=0, \\
\int d \mathbf{c}_{1} \int d \mathbf{c}_{2} c_{1 i} c_{2 i} \bar{T}\left(\mathbf{c}_{1}, \mathbf{c}_{2}\right) \chi_{H}\left(\mathbf{c}_{1}\right) \chi_{H}\left(\mathbf{c}_{2}\right)=\xi^{2},
\end{array}
$$

we can see that

$$
\begin{array}{r}
\int d \mathbf{l}_{12} \int d \mathbf{c}_{1} \int d \mathbf{c}_{2} H\left(\mathbf{l}_{12}, \mathbf{c}_{1}, \mathbf{c}_{2}\right)=0, \\
\int d \mathbf{l}_{12} \int d \mathbf{c}_{1} \int d \mathbf{c}_{2} c_{1 i} H\left(\mathbf{l}_{12}, \mathbf{c}_{1}, \mathbf{c}_{2}\right)=0, \\
\int d \mathbf{l}_{12} \int d \mathbf{c}_{1} \int d \mathbf{c}_{2} c_{1 i} c_{2 i} H\left(\mathbf{l}_{12}, \mathbf{c}_{1}, \mathbf{c}_{2}\right)=0 .
\end{array}
$$

If we multiply Eq. (4.2) by $\delta F\left(\mathbf{l}^{\prime}, \mathbf{c}^{\prime}, s^{\prime}\right)$, with $s^{\prime}<s$ and we average, we obtain

$$
\left[\frac{\partial}{\partial s}-\Lambda(\mathbf{c})+\mathbf{c} \cdot \frac{\partial}{\partial \mathbf{l}}\right]\left\langle\delta F(\mathbf{l}, \mathbf{c}, s) \delta F\left(\mathbf{l}^{\prime}, \mathbf{c}^{\prime}, s^{\prime}\right)\right\rangle_{H}=\left\langle R(\mathbf{l}, \mathbf{c}, s) \delta F\left(\mathbf{l}^{\prime}, \mathbf{c}^{\prime}, s^{\prime}\right)\right\rangle_{H} .
$$

We can easily see that

$$
\left\langle\delta F(\mathbf{l}, \mathbf{c}, s) \delta F\left(\mathbf{l}^{\prime}, \mathbf{c}^{\prime}, s^{\prime}\right\rangle_{H}=\frac{1}{n_{H} \ell^{d}} h_{H}\left(\mathbf{l}-\mathbf{l}^{\prime}, \mathbf{c}, \mathbf{c}^{\prime}, s-s^{\prime}\right) .\right.
$$

Now, if we compare Eqs. (2.39) and (4.13), we obtain that

$$
\left\langle R(\mathbf{l}, \mathbf{c}, s) \delta F\left(\mathbf{l}^{\prime}, \mathbf{c}^{\prime}, s^{\prime}\right)\right\rangle_{H}=0, \quad s>s^{\prime} .
$$


In the remaining of this section, we shall write the Boltzmann-Langevin equation together with noise properties in Fourier space. This will prove useful for the subsequent analysis. Let us introduce the Fourier component of a function of the position variable as

$$
\tilde{f}(\mathbf{k})=\int d \mathbf{l} e^{-i \mathbf{k} \cdot \mathbf{l}} f(\mathbf{l}), \quad f(\mathbf{l})=\frac{1}{\tilde{V}} \sum_{\mathbf{k}} e^{i \mathbf{k} \cdot \mathbf{l}} \tilde{f}(\mathbf{k}),
$$

where $\tilde{V}=\frac{V}{\ell^{d}}$ is the volume in units of the mean free path. The equation for $\delta \tilde{F}(\mathbf{k}, \mathbf{c}, s)$ is then

$$
\left[\frac{\partial}{\partial s}-\Lambda(\mathbf{k}, \mathbf{c})\right] \delta \tilde{F}(\mathbf{k}, \mathbf{c}, s)=\tilde{R}(\mathbf{k}, \mathbf{c}, s)
$$

where we have introduced the operator

$$
\Lambda(\mathbf{k}, \mathbf{c})=\Lambda(\mathbf{c})-i \mathbf{k} \cdot \mathbf{c}
$$

The Fourier transform of the noise, $\tilde{R}(\mathbf{k}, \mathbf{c}, s)$ obeys

$$
\begin{aligned}
\left\langle\tilde{R}\left(\mathbf{k}_{1}, \mathbf{c}_{1}, s_{1}\right)\right\rangle_{H} & =0, \\
\left\langle\tilde{R}\left(\mathbf{k}_{1}, \mathbf{c}_{1}, s_{1}\right) \delta \tilde{F}\left(\mathbf{k}_{2}, \mathbf{c}_{2}, s_{2}\right)\right\rangle_{H} & =0, \quad s_{1}>s_{2},
\end{aligned}
$$

and

$$
\left\langle\tilde{R}\left(\mathbf{k}_{1}, \mathbf{c}_{1}, s_{1}\right) \tilde{R}\left(\mathbf{k}_{2}, \mathbf{c}_{2}, s_{2}\right)\right\rangle_{H}=H\left(\mathbf{k}_{1}, \mathbf{k}_{2}, \mathbf{c}_{1}, \mathbf{c}_{2}\right) \delta\left(s_{1}-s_{2}\right)
$$

where

$$
H\left(\mathbf{k}_{1}, \mathbf{k}_{2}, \mathbf{c}_{1}, \mathbf{c}_{2}\right)=\frac{\tilde{V}^{2}}{N}\left[\Gamma\left(\mathbf{c}_{1}, \mathbf{c}_{2}\right) \delta\left(\mathbf{k}_{1}+\mathbf{k}_{2}\right)-\tilde{\xi}^{2} \frac{\partial}{\partial \mathbf{c}_{1}} \cdot \frac{\partial}{\partial \mathbf{c}_{2}} \chi_{H}\left(c_{1}\right) \chi_{H}\left(c_{2}\right) \delta\left(\mathbf{k}_{1}\right) \delta\left(\mathbf{k}_{2}\right)\right]
$$

which completes the calculation of the noise variance within the Langevin description.

\section{Fluctuations of the total energy}

As a first application of the Boltzmann-Langevin equation, let us derive a Langevin equation for the total energy. This calculation has been done using the correlation function formalism, and the result was compared to results of Direct simulation Monte Carlo method, obtaining a very good agreement for all the values of the coefficient $\alpha$. Now, we will show that, from the BoltzmannLangevin equation it is possible to deduce an equation for the total energy that gives the same results for the fluctuations.

We can write the energy fluctuations, in terms of the microscopic density, as

$$
\begin{aligned}
\delta E(t) & =\int d \mathbf{v} \int d \mathbf{r} \frac{1}{2} m v^{2}\left[F_{1}(x, t)-f_{H}(\mathbf{v})\right] \\
& =\frac{m v_{H}^{2} \ell^{d} n_{H}}{2} \int d \mathbf{c} c^{2} \int d \mathbf{l} \delta F(\mathbf{l}, \mathbf{c}, s) \\
& =\frac{m v_{H}^{2}}{2} \ell^{d} n_{H} \int d \mathbf{c} c^{2} \delta \tilde{F}(\mathbf{k}=\mathbf{0}, \mathbf{c}, s) .
\end{aligned}
$$


The dimensionless field $\delta \varepsilon(s)$ is defined by

$$
\delta \varepsilon(s)=\frac{\delta E(t)}{\langle E(t)\rangle_{H}}=\frac{1}{\tilde{V}} \int d \mathbf{c} \frac{2 c^{2}}{d} \delta \tilde{F}(\mathbf{k}=\mathbf{0}, \mathbf{c}, s),
$$

where $\langle E(t)\rangle_{H}$ is the total mean energy. We can identify $\delta \varepsilon(s)$ with the scalar product

$$
\delta \varepsilon(s)=\frac{2}{\tilde{V}}\left\langle\bar{\xi}_{3} \mid \delta \tilde{F}(\mathbf{k}=\mathbf{0}, \mathbf{c}, s)\right\rangle .
$$

If we consider now that $\bar{\xi}_{3}$ is approximately an eigenfunction of $\Lambda(\mathbf{c})$ with eigenvalue $\lambda_{3}=-\frac{3}{2} \zeta_{0}$, using the Boltzmann-Langevin equation we can write

$$
\left[\frac{d}{d s}+\frac{3}{2} \zeta_{0}\right] \delta \varepsilon(s)=R_{\varepsilon}(s)
$$

with

$$
R_{\varepsilon}(s)=\frac{2}{\tilde{V}}\left\langle\bar{\xi}_{3} \mid \tilde{R}(\mathbf{k}=\mathbf{0}, \mathbf{c}, s)\right\rangle .
$$

The next step is to find the properties of the noise term, $R_{\varepsilon}(s)$, making use of the properties of the noise of the Boltzmann-Langevin equation. From Eq. (4.20) it is clear that

$$
\left\langle R_{\varepsilon}(s) \delta \varepsilon\left(s^{\prime}\right)\right\rangle_{H}=0, \quad s>s^{\prime} .
$$

The correlation function of the noise is

$$
\begin{aligned}
\left\langle R_{\varepsilon}\left(s_{1}\right) R_{\varepsilon}\left(s_{2}\right)\right\rangle_{H} & =\frac{4}{\tilde{V}^{2}} \int d \mathbf{c}_{1} \int d \mathbf{c}_{2} \chi_{H}\left(c_{1}\right)^{-1} \chi_{H}\left(c_{2}\right)^{-1} \bar{\xi}_{3}\left(\mathbf{c}_{1}\right) \bar{\xi}_{3}\left(\mathbf{c}_{2}\right) H\left(\mathbf{l}_{12}, \mathbf{c}_{1}, \mathbf{c}_{2}\right) \delta\left(s_{1}-s_{2}\right) \\
& =\frac{4}{N} \delta\left(s_{1}-s_{2}\right)\left[\left\langle\bar{\xi}_{3}\left(\mathbf{c}_{1}\right) \bar{\xi}_{3}\left(\mathbf{c}_{2}\right) \mid \bar{T}\left(\mathbf{c}_{1}, \mathbf{c}_{2}\right) \chi_{H}\left(c_{1}\right) \chi_{H}\left(c_{2}\right)\right\rangle\right. \\
& \left.-\left\langle\bar{\xi}_{3}\left(\mathbf{c}_{1}\right) \bar{\xi}_{3}\left(\mathbf{c}_{2}\right) \mid\left(\Lambda\left(\mathbf{c}_{1}\right)+\Lambda\left(\mathbf{c}_{2}\right)\right) \chi_{H}\left(c_{1}\right) \delta\left(\mathbf{c}_{12}\right)\right\rangle\right]=A_{\varepsilon} \delta\left(s_{1}-s_{2}\right),
\end{aligned}
$$

where we have made use of Eq. (4.22) and Eq. (2.42). The calculation is now straightforward although lengthy. Taking into account the results obtained in [14], we have, in first order in Sonine polynomials that

$$
\left\langle\bar{\xi}_{3}\left(\mathbf{c}_{1}\right) \bar{\xi}_{3}\left(\mathbf{c}_{2}\right) \mid \bar{T}\left(\mathbf{c}_{1}, \mathbf{c}_{2}\right) \chi_{H}\left(c_{1}\right) \chi_{H}\left(c_{2}\right)\right\rangle=3 \zeta_{0} a_{33},
$$

with

$$
a_{33}=\frac{\left.-15+7 d+14 d^{2}-3(-9+d(9+2 d)) \alpha+30(1+d) \alpha^{2}-6(9+d) \alpha^{3}\right)}{9 d(-19+2 d(-7+3 \alpha)+3 \alpha(9+2(-1+\alpha) \alpha))} .
$$

The other term can be easily evaluated in our approximation

$$
\begin{aligned}
\left\langle\bar{\xi}_{3}\left(\mathbf{c}_{1}\right) \bar{\xi}_{3}\left(\mathbf{c}_{2}\right) \mid\left(\Lambda\left(\mathbf{c}_{1}\right)+\Lambda\left(\mathbf{c}_{2}\right)\right) \chi_{H}\left(c_{1}\right) \delta\left(\mathbf{c}_{12}\right)\right\rangle & \simeq-3 \zeta_{0} \int d \mathbf{c}\left(\frac{c^{2}}{d}-\frac{1}{6}\right)^{2} \chi_{H}(c) \\
& =-3 \zeta_{0}\left(-\frac{5}{36}+\frac{d+2}{4 d}\left(1+a_{2}\right)\right)
\end{aligned}
$$


Finally, we can identify $A_{\varepsilon}$ to be

$$
A_{\varepsilon}=\frac{12}{N} \zeta_{0}\left(a_{33}-\frac{5}{36}+\frac{d+2}{4 d}\left(1+a_{2}\right)\right) .
$$

With the properties we have about the noise $R_{\varepsilon}(s)$, it is easy to calculate the correlation function of $\delta \varepsilon$. The solution of Eq. (5.4) can be formally written as

$$
\delta \varepsilon(s)=e^{-\frac{3}{2} \zeta_{0} s} \delta \varepsilon(0)+\int_{0}^{s} d s^{\prime} e^{-\frac{3}{2} \zeta_{0}\left(s-s^{\prime}\right)} R_{\varepsilon}\left(s^{\prime}\right) \stackrel{s \gg 1}{\longrightarrow} \int_{0}^{s} d s^{\prime} e^{-\frac{3}{2} \zeta_{0}\left(s-s^{\prime}\right)} R_{\varepsilon}\left(s^{\prime}\right) .
$$

So, we can evaluate

$$
\left\langle\delta \varepsilon(s)^{2}\right\rangle_{H}=\int_{0}^{s} d s_{1} \int_{0}^{s} d s_{2} e^{-\frac{3}{2} \zeta_{0}\left[\left(s-s_{1}\right)+\left(s-s_{2}\right)\right]}\left\langle R_{\varepsilon}\left(s_{1}\right) R_{\varepsilon}\left(s_{2}\right)\right\rangle_{H}=\int_{0}^{s} d s_{1} e^{-3 \zeta_{0}\left(s-s_{1}\right)} A_{\varepsilon} \stackrel{s \gg 1}{\longrightarrow} \frac{A_{\varepsilon}}{3 \zeta_{0}} .
$$

In Fig. 1, we show the comparison between the theoretical result (solid line) and the DSMC method results (circles and squares) for a two-dimensional system, as a function of the coefficient of normal restitution. As can be seen, the agreement is satisfactory for all the range of values of the inelasticity. In the limit, $\alpha \rightarrow 1^{-}$, we find the non-trivial result $\left\langle\delta \varepsilon^{2}\right\rangle_{H} \rightarrow \frac{d}{3}$, while in the free cooling state, this quantity vanishes [7]. We emphasize that the elastic limit is singular: the behaviour for elastic systems with $\alpha=1$ is not approached by taking the quasi-elastic limit $\alpha \rightarrow 1^{-}$.

We can also evaluate the two-time correlation function for the fluctuations of the total energy. If we multiply in Eq. (5.4) by $\delta \varepsilon\left(s^{\prime}\right)$, for $s^{\prime}<s$, and taking into account that the noise is not correlated with the distribution function for a time smaller, we obtain that

$$
\left\langle\delta \varepsilon(s) \delta \varepsilon\left(s^{\prime}\right)\right\rangle_{H}=\left\langle\delta \varepsilon\left(s^{\prime}\right)^{2}\right\rangle_{H} e^{-\frac{3}{2} \zeta_{0}\left(s-s^{\prime}\right)} .
$$

Then, the decay of the two-time correlation function is the same as we have found for a homogeneous perturbation of the total energy. As expected, the amplitude of the fluctuations coincides with the one obtained in [14] from the equations for the correlation functions.

\section{The fluctuating transverse velocity}

The objective in this section is to derive a fluctuating equation for the transverse velocity field, $\mathbf{w}_{\perp}(\mathbf{k}, s)$. The reason to consider this field is that its equation is decoupled from the equations for the other hydrodynamic fields [31], and it can be derived exactly in the hydrodynamic limit with the knowledge we have about the spectrum of the linearized Boltzmann-Fokker-Planck operator, i.e. we do not need the approximation corresponding to the third eigenfunction.

Mathematically, the transverse velocity is defined in the following way: Let us consider the $d$-dimensional vector $\mathbf{k}$ which belongs to the space $\Re^{d}$. This space can be spanned by the base 


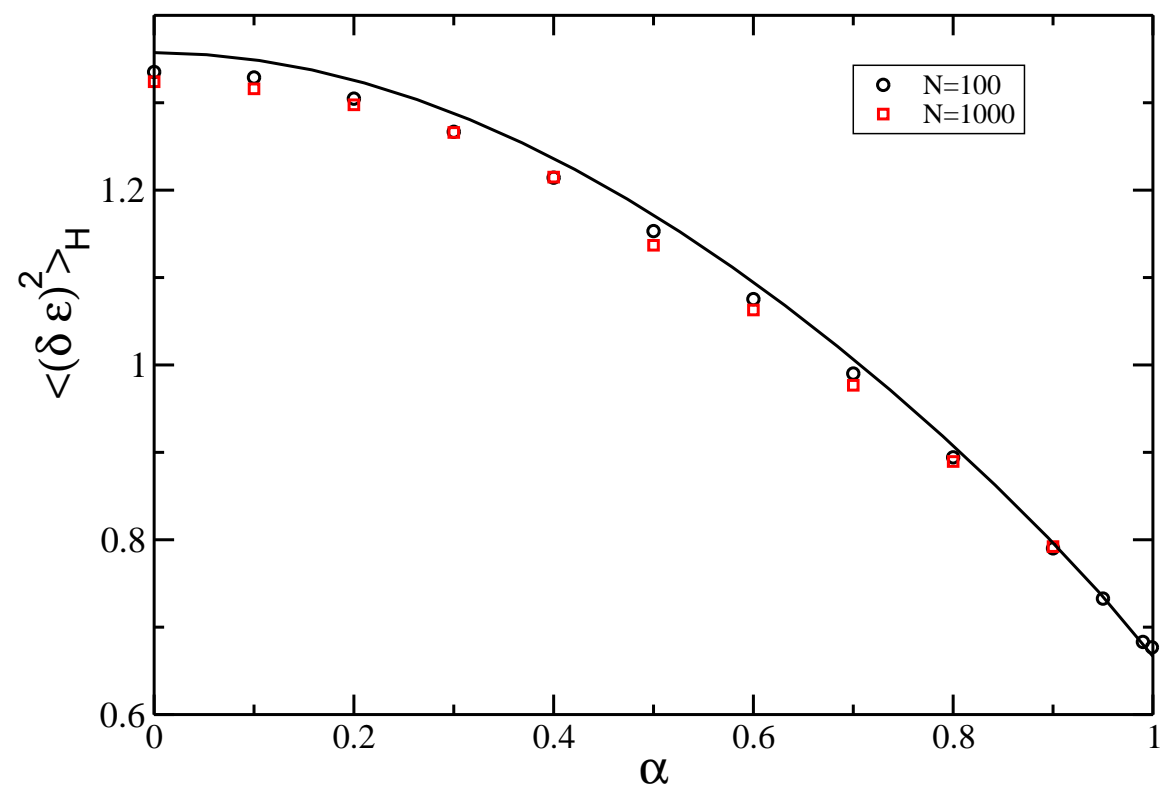

Figure 1: Second moment of the fluctuations of the total energy as a function of the restitution coefficient $\alpha$. The solid line is the theoretical prediction and symbols are the two-dimensional Monte Carlo simulation results of Ref. [32].

$\{\hat{\mathbf{k}}\} \bigcup\left\{\hat{\mathbf{k}}_{\perp}^{i}\right\}_{i=1}^{d-1}$ where $\hat{\mathbf{k}}$ is a unitary vector parallel to $\mathbf{k}$ and $\left\{\hat{\mathbf{k}}_{\perp}^{i}\right\}_{i=1}^{d-1}$ are $(d-1)$ unitary vectors orthogonal to $\mathrm{k}$. The transverse velocity is defined as

$$
w_{\perp i}(\mathbf{k}, s)=\int d \mathbf{c}\left(\mathbf{c} \cdot \hat{\mathbf{k}}_{\perp}^{i}\right) \delta \tilde{F}(\mathbf{k}, \mathbf{c}, s), \quad i=1, \ldots, d-1 .
$$

For the subsequent analysis, it is convenient to introduce the following projectors

$$
\begin{gathered}
P^{(i)} f(\mathbf{c}) \equiv\left\langle\bar{\xi}_{2 \perp i}(\mathbf{c}) \mid f(\mathbf{c})\right\rangle \xi_{2 \perp i}(\mathbf{c}), \\
Q^{(i)} f(\mathbf{c}) \equiv\left(1-P^{(i)}\right) f(\mathbf{c}) .
\end{gathered}
$$

Then, if we apply $P^{(i)}$ to $\delta \tilde{F}$ we obtain

$$
P^{(i)} \delta \tilde{F}(\mathbf{k}, \mathbf{c}, s)=w_{\perp i}(\mathbf{k}, s) \xi_{2 \perp i}(\mathbf{c}),
$$

and the transverse velocity is the component of $\delta \tilde{F}(\mathbf{k}, \mathbf{c}, s)$ in the subspace generated by $\xi_{2 \perp i}(\mathbf{c})$.

In order to obtain an equation for $\mathbf{w}_{\perp}$, we apply the projectors $P$ and $Q$ (for simplicity in the notation we skip the super-index) to the Langevin equation (4.17)

$$
\left[\frac{\partial}{\partial s}-P \Lambda(\mathbf{k}, \mathbf{c})\right] P \delta \tilde{F}(\mathbf{k}, \mathbf{c}, s)=P \tilde{R}(\mathbf{k}, \mathbf{c}, s)-P i(\mathbf{k} \cdot \mathbf{c}) Q \delta \tilde{F}(\mathbf{k}, \mathbf{c}, s)
$$




$$
\left[\frac{\partial}{\partial s}-Q \Lambda(\mathbf{k}, \mathbf{c})\right] Q \delta \tilde{F}(\mathbf{k}, \mathbf{c}, s)=Q \tilde{R}(\mathbf{k}, \mathbf{c}, s)-Q i(\mathbf{k} \cdot \mathbf{c}) P \delta \tilde{F}(\mathbf{k}, \mathbf{c}, s) .
$$

Now, let us solve equation (6.6) formally

$$
\begin{aligned}
Q \delta \tilde{F}(\mathbf{k}, \mathbf{c}, s) & =e^{Q \Lambda(\mathbf{k}, \mathbf{c}) s} Q \delta \tilde{F}(\mathbf{k}, \mathbf{c}, 0) \\
& +\int_{0}^{s} d s^{\prime} e^{Q \Lambda(\mathbf{k}, \mathbf{c})\left(s-s^{\prime}\right)}\left[Q \tilde{R}\left(\mathbf{k}, \mathbf{c}, s^{\prime}\right)-Q i(\mathbf{k} \cdot \mathbf{c}) P \delta \tilde{F}\left(\mathbf{k}, \mathbf{c}, s^{\prime}\right)\right] .
\end{aligned}
$$

Restricting ourselves to initial conditions with $Q \delta \tilde{F}(\mathbf{k}, \mathbf{c}, s)=0$, and by substituting Eq. (6.7) in Eq. (6.5), we obtain a closed equation for $P \delta \tilde{F}(\mathbf{k}, \mathbf{c}, s)$

$$
\begin{aligned}
{\left[\frac{\partial}{\partial s}-P \Lambda(\mathbf{k}, \mathbf{c})\right] P \delta \tilde{F}(\mathbf{k}, \mathbf{c}, s) } & +P(\mathbf{k} \cdot \mathbf{c}) \int_{0}^{s} d s^{\prime} e^{Q \Lambda(\mathbf{k}, \mathbf{c})\left(s-s^{\prime}\right)} Q(\mathbf{k} \cdot \mathbf{c}) P \delta \tilde{F}\left(\mathbf{k}, \mathbf{c}, s^{\prime}\right) \\
& =P \tilde{R}(\mathbf{k}, \mathbf{c}, s) \\
& -P i(\mathbf{k} \cdot \mathbf{c}) \int_{0}^{s} d s^{\prime} e^{Q \Lambda(\mathbf{k}, \mathbf{c})\left(s-s^{\prime}\right)} Q \tilde{R}\left(\mathbf{k}, \mathbf{c}, s^{\prime}\right)
\end{aligned}
$$

In Appendix B it is shown that, in the hydrodynamic limit, i.e. to second order in $k$ and in the long time limit, equation (6.8) reduces to the following equation for the transverse velocity

$$
\left[\frac{\partial}{\partial s}+\tilde{\eta} k^{2}\right] w_{\perp}(\mathbf{k}, s)=R_{w}(\mathbf{k}, s) .
$$

The coefficient $\tilde{\eta}$ is the shear viscosity given by

$$
\tilde{\eta}=\int_{0}^{\infty} d s \int d \mathbf{c} c_{x} c_{y} e^{\Lambda(\mathbf{c}) s} c_{x} \xi_{2, y}(\mathbf{c})=-\int d \mathbf{c} c_{x} c_{y} \Lambda(\mathbf{c})^{-1} c_{x} \xi_{2, y}(\mathbf{c}),
$$

which agrees with the expression obtained in [15] by the Chapman-Enskog method, and $R_{w}(\mathbf{k}, s)$ is a noise term which can be decomposed as

$$
R_{w}(\mathbf{k}, s)=S(\mathbf{k}, s)+\Pi(\mathbf{k}, s) .
$$

The term $S(\mathbf{k}, s)$ comes from the thermostat (which does not conserve momentum locally), and the second term, $\Pi(\mathbf{k}, s)$, is the fluctuating part of the pressure tensor. Their microscopic expressions in terms of the noise of the Boltzmann-Langevin equation are

$$
S(\mathbf{k}, s)=\int d \mathbf{c}\left(\hat{\mathbf{k}}_{\perp} \cdot \mathbf{c}\right) \tilde{R}(\mathbf{k}, \mathbf{c}, s),
$$

and

$$
\Pi(\mathbf{k}, s)=-i k \int_{0}^{s} d s^{\prime} \int d \mathbf{c}(\hat{\mathbf{k}} \cdot \mathbf{c})\left(\hat{\mathbf{k}}_{\perp} \cdot \mathbf{c}\right) e^{Q \Lambda(\mathbf{k}, \mathbf{c})\left(s-s^{\prime}\right)} Q \tilde{R}\left(\mathbf{k}, \mathbf{c}, s^{\prime}\right) .
$$

Of course, due to the fact that $\langle\tilde{R}(\mathbf{k}, \mathbf{c}, s)\rangle_{H}=0$, the mean value of the noise vanishes

$$
\left\langle R_{w}(\mathbf{k}, s)\right\rangle_{H}=0 .
$$


The autocorrelation function of the noise is evaluated in Appendix C. Due to symmetry considerations, there are only correlations between the $\mathrm{k}$ and $-\mathrm{k}$ components, which yields

$$
\begin{aligned}
\left\langle R_{w}\left(\mathbf{k}, s_{1}\right) R_{w}\left(-\mathbf{k}, s_{2}\right)\right\rangle_{H} & =\frac{\tilde{V}^{2}}{N}\left[\xi^{2} \delta\left(s_{1}-s_{2}\right)+k^{2} C_{x y}\left(s_{2}-s_{1}\right)\right] \\
& +\left\langle S\left(\mathbf{k}, s_{1}\right) \Pi\left(-\mathbf{k}, s_{2}\right)\right\rangle_{H}, \quad s_{1}<s_{2} .
\end{aligned}
$$

The first term is the expected zeroth order term which comes from the heating. The Dirac delta function is an exact consequence of the fact that the external noise (the heating) is white. The function $C_{x y}\left(s_{2}-s_{1}\right)$ reads

$$
C_{x y}\left(s_{2}-s_{1}\right)=\int d \mathbf{c}_{1} \int d \mathbf{c}_{2} c_{1 x} c_{1 y} c_{2 x} c_{2 y} e^{\Lambda\left(\mathbf{c}_{2}\right)\left(s_{2}-s_{1}\right)} \phi_{H}\left(\mathbf{c}_{1}, \mathbf{c}_{2}\right) .
$$

Here, we have introduced the function $\phi_{H}\left(\mathbf{c}_{1}, \mathbf{c}_{2}\right)$ as the space integral of the correlation function $h_{H}\left(\mathbf{l}, \mathbf{c}_{1}, \mathbf{c}_{2}\right)$

$$
\phi_{H}\left(\mathbf{c}_{1}, \mathbf{c}_{2}\right)=\int d \mathbf{l} h_{H}\left(\mathbf{l}, \mathbf{c}_{1}, \mathbf{c}_{2}\right)=\chi_{H}\left(c_{1}\right) \delta\left(\mathbf{c}_{12}\right)+\chi_{2}\left(\mathbf{c}_{1}, \mathbf{c}_{2}\right),
$$

where $\chi_{2}\left(\mathbf{c}_{1}, \mathbf{c}_{2}\right)=\int d \mathbf{l} g_{H}\left(\mathbf{l}, \mathbf{c}_{1}, \mathbf{c}_{2}\right)$ and $g_{H}$ is the dimensionless correlation function defined in (2.36). The equation for $\phi_{H}$ can easily be obtained by integration over space variable in Eq. (2.41), which gives

$$
\left[\Lambda\left(\mathbf{c}_{1}\right)+\Lambda\left(\mathbf{c}_{2}\right)\right] \phi_{H}\left(\mathbf{c}_{1}, \mathbf{c}_{2}\right)=-\Gamma\left(\mathbf{c}_{1}, \mathbf{c}_{2}\right)+\xi^{2} \frac{\partial}{\partial \mathbf{c}_{1}} \cdot \frac{\partial}{\partial \mathbf{c}_{2}} \chi_{H}\left(c_{1}\right) \chi_{H}\left(c_{2}\right)
$$

As discussed in Appendix C, $C_{x y}$ can be physically interpreted as the autocorrelation function of the global quantity $\sum_{i=1}^{N} V_{x}(t) V_{y}(t)$. In the elastic case, this correlation function is related to the shear viscosity but it is not the case for granular systems [1]. The formula for the correlation $\left\langle S\left(\mathbf{k}, s_{1}\right) \Pi\left(-\mathbf{k}, s_{2}\right)\right\rangle_{H}$ is given in Appendix $\mathbf{C}$, and it does not seem to admit a simple physical interpretation. As the two correlation functions, $C_{x y}(s)$ and $\left\langle S\left(\mathbf{k}, s_{1}\right) \Pi\left(-\mathbf{k}, s_{2}\right)\right\rangle_{H}$, decay with the kinetic modes and, in the $k \rightarrow 0$ limit, the fluctuating velocity is expected to be frozen (its time evolution is given by the null eigenvalue, Eq. (6.9)), we can consider that they are proportional to a Dirac delta function in time, and we have

$$
\begin{aligned}
& \frac{\tilde{V}^{2}}{N} k^{2} C_{x y}\left(s_{2}-s_{1}\right)+\left\langle S\left(\mathbf{k}, s_{1}\right) \Pi\left(-\mathbf{k}, s_{2}\right)\right\rangle_{H} \\
& \rightarrow 2\left[\frac{\tilde{V}^{2}}{N} k^{2} \int_{0}^{\infty} d s C_{x y}(s)+\int_{0}^{\infty} d s\langle S(\mathbf{k}, 0) \Pi(-\mathbf{k}, s)\rangle_{H}\right] \delta\left(s_{1}-s_{2}\right) .
\end{aligned}
$$

Note that this is in contrast with the free cooling case where the noise can not be considered to be white [6]. In this case, the equation for the transverse velocity (rescaled with the thermal velocity) contains a term of order zero in $k$ which is proportional to the cooling rate. Then, even in the 
$k \rightarrow 0$ limit, the velocity is not frozen on the kinetic scale. In Appendix D the second integral of equation (6.19) is evaluated obtaining

$$
\int_{0}^{\infty} d s\langle S(\mathbf{k}, 0) \Pi(-\mathbf{k}, s)\rangle_{H} \rightarrow \frac{\tilde{V}^{2}}{N} k^{2} \int d \mathbf{c}_{1} \int d \mathbf{c}_{2} c_{1 x} c_{2 x} c_{2 y} \Lambda\left(\mathbf{c}_{2}\right)^{-1} c_{2 y} \phi_{H}\left(\mathbf{c}_{1}, \mathbf{c}_{2}\right),
$$

which is valid in the hydrodynamic limit. If we substitute $\phi_{H}\left(\mathbf{c}_{1}, \mathbf{c}_{2}\right)$ by its expression in terms of the one- and two-particle distribution function, Eq. (6.17), we obtain (see Appendix E) that the one-particle contribution vanishes and the correlation function can be written in terms of the two-particle velocity correlation function, $\chi_{2}\left(\mathbf{c}_{1}, \mathbf{c}_{2}\right)$

$$
\begin{aligned}
\left\langle R_{w}\left(\mathbf{k}, s_{1}\right) R_{w}\left(-\mathbf{k}, s_{2}\right)\right\rangle_{H} & \\
=\frac{\tilde{V}^{2}}{N} \delta\left(s_{1}-s_{2}\right)\left\{\xi^{2}+\right. & 2 k^{2}\left[-\int d \mathbf{c}_{1} \int d \mathbf{c}_{2} c_{1 x} c_{1 y} c_{2 x} c_{2 y} \Lambda\left(\mathbf{c}_{2}\right)^{-1} \chi_{2}\left(\mathbf{c}_{1}, \mathbf{c}_{2}\right)\right. \\
& \left.\left.+\int d \mathbf{c}_{1} \int d \mathbf{c}_{2} c_{1 x} c_{2 x} c_{2 y} \Lambda\left(\mathbf{c}_{2}\right)^{-1} c_{2 y} \chi_{2}\left(\mathbf{c}_{1}, \mathbf{c}_{2}\right)\right]\right\}
\end{aligned}
$$

As can be seen, the $k^{2}$ term has no relation, a priori, with the shear viscosity, Eq. (6.10), i.e. there is a priori no fluctuation-dissipation relation as that assumed in [31]. However, we now show that, under additional hypotheses (that in principle are not restricted to the elastic limit), the aforementioned term reduces to the shear viscosity. Let us assume that the most important contribution of the two-particle velocity correlation function, $\chi_{2}\left(\mathbf{c}_{1}, \mathbf{c}_{2}\right)$, is the hydrodynamic part, i.e. we assume

$$
\chi_{2}\left(\mathbf{c}_{1}, \mathbf{c}_{2}\right) \simeq \sum_{\beta=1}^{d+2} \sum_{\beta^{\prime}=1}^{d+2} a_{\beta, \beta^{\prime}} \xi_{\beta}\left(\mathbf{c}_{1}\right) \xi_{\beta^{\prime}}\left(\mathbf{c}_{2}\right) .
$$

This assumption was already made in [14] where the coefficients $a_{\beta, \beta^{\prime}}$ were evaluated to calculate the total energy fluctuations. We emphasize that it led to an excellent agreement between analytical predictions and numerical data (Monte Carlo) for energy fluctuations, for all the values of the inelasticity [14]. In this approximation, the first integral in (6.21) vanishes because $c_{x} c_{y} \chi_{H}(c)$ is orthogonal to the hydrodynamic modes. The second term is

$$
\begin{array}{r}
\int d \mathbf{c}_{1} \int d \mathbf{c}_{2} c_{1 x} c_{2 x} c_{2 y} \Lambda\left(\mathbf{c}_{2}\right)^{-1} c_{2 y} \sum_{\beta=1}^{d+2} \sum_{\beta^{\prime}=1}^{d+2} a_{\beta, \beta^{\prime}} \xi_{\beta}\left(\mathbf{c}_{1}\right) \xi_{\beta^{\prime}}\left(\mathbf{c}_{2}\right) \\
=a_{2 x, 2 x} \int d \mathbf{c}_{1} c_{1 x} \xi_{2 x}\left(\mathbf{c}_{1}\right) \int d \mathbf{c}_{2} c_{2 x} c_{2 y} \Lambda\left(\mathbf{c}_{2}\right)^{-1} c_{2 y} \xi_{2 x}\left(\mathbf{c}_{2}\right)
\end{array}
$$

where, for symmetry considerations, the only term that remains is the one associated with $\beta=$ $\beta^{\prime}=2$. If we use now that $a_{2 i 2 i}=-1 / 2$ (see the reference [14]), and the formula for the shear viscosity, Eq. (6.10), we finally have

$$
\left\langle R_{w}\left(\mathbf{k}, s_{1}\right) R_{w}\left(-\mathbf{k}, s_{2}\right)=\frac{\tilde{V}^{2}}{N} \delta\left(s_{1}-s_{2}\right)\left(\xi^{2}+\tilde{\eta} k^{2}\right) .\right.
$$


Then, in the hydrodynamic limit and assuming that the two-particle velocity correlation function, $\chi_{2}\left(\mathbf{c}_{1}, \mathbf{c}_{2}\right)$, has only components in the hydrodynamic subspace, the correlation function of the noise reduces to the one introduced phenomenologically in [31], not only in the elastic limit, but for arbitrary inelasticity. Although we have no direct proof of the accuracy of approximation (6.22), we note that it is backed up by numerical data, see e.g. [14].

As an application of Eq. (6.9), let us calculate the two-time correlation for the transverse velocity field. Proceeding as in Sec. 5., the two-time correlation function can be calculated, obtaining

$$
\left\langle w_{\perp}(\mathbf{k}, s) w_{\perp}\left(-\mathbf{k}, s^{\prime}\right)\right\rangle_{H}=\frac{\tilde{V}^{2}}{N} \frac{\xi^{2}+\tilde{\eta} k^{2}}{2 \tilde{\eta} k^{2}} e^{-\tilde{\eta} k^{2}\left(s-s^{\prime}\right)} .
$$

As seen, the correlation function decays as a linear macroscopic perturbation, with an amplitude which is a function of the heating and the shear viscosity.

\section{Conclusions}

In this work, we have presented a theory of fluctuations for a dilute gas heated by a stochastic thermostat in the stationary state. The starting point has been the Boltzmann-Langevin equation: a stochastic equation for the fluctuating distribution function. This equation has the same form as the linearized Boltzmann equation but adding a noise term. The properties of the noise are determined enforcing consistency with the equations for the correlation functions, for both the one-time and the two-time correlation functions. We consider that the noise is white as it is the case for molecular fluids. From this equation it would be possible to construct a set of fluctuating hydrodynamic equations, and from them to evaluate the one-time and two-time correlation functions of the fluctuating fields. We have focused on the study of the simplest two fluctuating quantities: the total energy and the transverse velocity, whose equations are decoupled. The results obtained for the energy fluctuations match up with the ones calculated earlier by means of the equation for the correlation function [14]. We have also deduced the decay of the correlations for the fluctuations, finding that they decay in the same way as a linear perturbation. These results have been successfully tested against the numerical results obtained by the Direct simulation Monte Carlo method for all the range of values of the coefficient of normal restitution $\alpha[14,32]$. For the transverse velocity field, some important differences have been found in comparison with the free cooling case $[5,6]$, where it was shown that the relevant Langevin noise is not white and that there is no a fluctuation-dissipation relation of the second kind (the amplitude of the noise is not related to the shear viscosity). Firstly, the noise has two parts, one coming from the thermostat and the other one coming from the fluctuating pressure tensor. Moreover, the noise can be considered to be white as the dynamics of the velocity is as slow as desired in the hydrodynamic (low $k$ ) limit. With regard to the validity of the fluctuation-dissipation relation in the heated case in the hydrodynamic limit, the amplitude of the noise is priori not related to the shear viscosity. However, considering that the two-particle velocity correlation function has only hydrodynamic modes -which seems a reasonable assumption- the coupling between the two noises somehow restores fluctuation-dissipation and we obtain the expression assumed in [31], with the actual inelastic shear viscosity. In principle, this rather surprising result is not limited to small inelasticity. 


\section{Acknowledgements}

We thank Emmanuel Trizac for useful discussions and for a careful reading of the manuscript. We also thank Paolo Visco for providing us with the Monte Carlo data of Fig. 1. This research was supported by the Ministerio de Educación y Ciencia (Spain) through Grant No. FIS2008-01339 (partially financed by FEDER funds).

\section{A Eigenvalue problem for $\Lambda$}

We consider here the eigenvalue problem for the homogeneous linear Boltzmann-Fokker-Planck operator $\Lambda$, defined in (2.38)

$$
\Lambda(\mathbf{c}) \xi_{\beta}(\mathbf{c})=\lambda_{\beta} \xi_{\beta}(\mathbf{c}) .
$$

We are interested in the eigenfunctions and eigenvalues associated with linear hydrodynamics and, to perform the analysis, similar techniques as in $[3,7,13]$ will be required.

Consider first the function

$$
\psi_{1}(\mathbf{c})=\chi_{H}(c)
$$

When the linearized operator $\Lambda$ acts on $\chi_{H}$, we have

$$
\Lambda\left(\mathbf{c}_{1}\right) \chi_{H}\left(\mathbf{c}_{1}\right)=\int d \mathbf{c}_{2} \bar{T}\left(\mathbf{c}_{2}, \mathbf{c}_{3}\right)\left(1+\mathcal{P}_{12}\right) \chi_{H}\left(c_{2}\right) \chi_{H}\left(c_{1}\right)+\frac{\xi^{2}}{2}\left(\frac{\partial}{\partial \mathbf{c}_{1}}\right)^{2} \chi_{H}\left(c_{1}\right) .
$$

Taking into account the equation for $\chi_{H}$, Ec. (2.29), we obtain the following relation

$$
\Lambda\left(\mathbf{c}_{1}\right) \psi_{1}\left(c_{1}\right)=-\frac{\xi^{2}}{2}\left(\frac{\partial}{\partial \mathbf{c}_{1}}\right)^{2} \chi_{H}\left(c_{1}\right) .
$$

Now, let us considerer the function

$$
\boldsymbol{\psi}_{2}(\mathbf{c})=-\frac{\partial}{\partial \mathbf{c}} \chi_{H}(c)
$$

Taking derivate in the equation obeyed by $\chi_{H}(\mathbf{c}-\mathbf{w})$ with respect to $\mathbf{w}$, and subsequently evaluating the result for $\mathbf{w}=0$, we obtain

$$
\Lambda\left(\mathbf{c}_{1}\right) \boldsymbol{\psi}_{2}\left(\mathbf{c}_{1}\right)=\mathbf{0}
$$

Finally, we will consider the function

$$
\psi_{3}(\mathbf{c})=\mathbf{c} \cdot \frac{\partial}{\partial \mathbf{c}} \chi_{H}(c) .
$$

From the equation obeyed by $\psi_{3}\left(\lambda \mathbf{c}_{1}\right)$, we can take derivate with respect to $\lambda$, and evaluate the result for $\lambda=1$. We arrive at an equation for $\psi_{3}\left(\mathbf{c}_{1}\right)$,

$$
\Lambda\left(\mathbf{c}_{1}\right) \psi_{3}\left(\mathbf{c}_{1}\right)=(d+3) \frac{\xi^{2}}{2}\left(\frac{\partial}{\partial \mathbf{c}_{1}}\right)^{2} \chi_{H}\left(c_{1}\right)
$$


From equations (A4), (A6) and (A8), we can identify two eigenfunctions of $\Lambda$. Making use of (A4) and (A8), it appears that

$$
\Lambda(\mathbf{c})\left(\frac{1}{3} \frac{\partial}{\partial \mathbf{c}} \cdot\left[\mathbf{c} \chi_{H}(c)\right]+\chi_{H}(c)\right)=0 .
$$

Hence, from Eqs. (A6) and (A9) we can conclude that the null eigenvalue is $(d+1)$-fold degenerate with the eigenfunctions

$$
\xi_{1}(\mathbf{c})=\frac{1}{3} \frac{\partial}{\partial \mathbf{c}} \cdot\left[\mathbf{c} \chi_{H}(c)\right]+\chi_{H}(c), \quad \boldsymbol{\xi}_{2}=-\frac{\partial}{\partial \mathbf{c}} \chi_{H}(c)
$$

\section{B Derivation of the transverse velocity field equation}

In this Appendix we derive the equation for the transverse velocity field, $w_{\perp}(\mathbf{k}, s)$, in the hydrodynamic limit. The starting point is equation (6.8)

$$
\begin{aligned}
{\left[\frac{\partial}{\partial s}-P \Lambda(\mathbf{k}, \mathbf{c})\right] P \delta \tilde{F}(\mathbf{k}, \mathbf{c}, s) } & +P(\mathbf{k} \cdot \mathbf{c}) \int_{0}^{s} d s^{\prime} e^{Q \Lambda(\mathbf{k}, \mathbf{c})\left(s-s^{\prime}\right)} Q(\mathbf{k} \cdot \mathbf{c}) P \delta \tilde{F}\left(\mathbf{k}, \mathbf{c}, s^{\prime}\right) \\
& =P \tilde{R}(\mathbf{k}, \mathbf{c}, s) \\
& -P i(\mathbf{k} \cdot \mathbf{c}) \int_{0}^{s} d s^{\prime} e^{Q \Lambda(\mathbf{k}, \mathbf{c})\left(s-s^{\prime}\right)} Q \tilde{R}\left(\mathbf{k}, \mathbf{c}, s^{\prime}\right) .
\end{aligned}
$$

Let us first consider the term $P \Lambda(\mathbf{k}, \mathbf{c}) P \delta \tilde{F}(\mathbf{k}, \mathbf{c}, s)$. As $\Lambda(\mathbf{c}) \xi_{2 \perp}(\mathbf{c})=0$ and $\int d \mathbf{c}\left(\hat{\mathbf{k}}_{\perp} \cdot \mathbf{c}\right)(\hat{\mathbf{k}}$. c) $\xi_{2 \perp}(\mathbf{c})=0$, we easily have

$$
P \Lambda(\mathbf{k}, \mathbf{c}) P \delta \tilde{F}(\mathbf{k}, \mathbf{c}, s)=0 .
$$

Let us evaluate the last term of the left-hand side of Eq. (B1). To second order in $k$, we have

$$
\begin{array}{r}
P(\mathbf{k} \cdot \mathbf{c}) \int_{0}^{s} d s^{\prime} e^{Q \Lambda(\mathbf{k}, \mathbf{c})\left(s-s^{\prime}\right)} Q(\mathbf{k} \cdot \mathbf{c}) P \delta \tilde{F}\left(\mathbf{k}, \mathbf{c}, s^{\prime}\right) \\
\simeq k^{2} \xi_{2 \perp}(\mathbf{c}) \int_{0}^{s} d s^{\prime} w_{\perp}\left(\mathbf{k}, s^{\prime}\right) \int d \mathbf{c}(\hat{\mathbf{k}} \cdot \mathbf{c})\left(\hat{\mathbf{k}}_{\perp} \cdot \mathbf{c}\right) e^{\Lambda(\mathbf{c})\left(s-s^{\prime}\right)} \hat{\mathbf{k}} \cdot \mathbf{c} \xi_{2 \perp}(\mathbf{c}) \\
=k^{2} \xi_{2 \perp}(\mathbf{c}) \int_{0}^{s} d s^{\prime} w_{\perp}\left(\mathbf{k}, s^{\prime}\right) G_{x y}\left(s-s^{\prime}\right)
\end{array}
$$

where we have introduced

$$
\begin{array}{r}
G_{x y}(s) \equiv \int d \mathbf{c}\left(\hat{\mathbf{k}}_{\perp} \cdot \mathbf{c}\right)(\hat{\mathbf{k}} \cdot \mathbf{c}) e^{\Lambda(\mathbf{c}) s}(\hat{\mathbf{k}} \cdot \mathbf{c}) \xi_{2 \perp}(\mathbf{c}) \\
=\int d \mathbf{c} c_{x} c_{y} e^{\Lambda(\mathbf{c}) s} c_{x} \xi_{2 y}(\mathbf{c}),
\end{array}
$$

and use has been made of the fact that the operator $\Lambda(\mathbf{c})$ is isotropic. In the hydrodynamic limit, the velocity evolves in a scale much slower that the scale in which the function $G_{x y}(s)$ decays. We then have

$$
\int_{0}^{s} d s^{\prime} w_{\perp}\left(\mathbf{k}, s^{\prime}\right) G_{x y}\left(s-s^{\prime}\right) \rightarrow \tilde{\eta} w_{\perp}(\mathbf{k}, s)
$$


where $\tilde{\eta}$ is the dimensionless shear viscosity

$$
\tilde{\eta}=\int_{0}^{\infty} d s G_{x y}(s)
$$

The noise terms are the last two terms of Eq. (B1)

$$
\begin{aligned}
P \tilde{R}(\mathbf{k}, \mathbf{c}, s) & =\xi_{2 \perp}(\mathbf{c}) \int d \mathbf{c}\left(\hat{\mathbf{k}}_{\perp} \cdot \mathbf{c}\right) \tilde{R}(\mathbf{k}, \mathbf{c}, s) \\
& =\xi_{2 \perp}(\mathbf{c}) S(\mathbf{k}, s)
\end{aligned}
$$

and

$$
\begin{aligned}
& P(i \mathbf{k} \cdot \mathbf{c}) \int_{0}^{s} d s^{\prime} e^{Q \Lambda(\mathbf{k}, \mathbf{c})\left(s-s^{\prime}\right)} Q \tilde{R}\left(\mathbf{k}, \mathbf{c}, s^{\prime}\right) \\
& =\xi_{2 \perp}(\mathbf{c}) i k \int d \mathbf{c}\left(\hat{\mathbf{k}}_{\perp} \cdot \mathbf{c}\right)(\hat{\mathbf{k}} \cdot \mathbf{c}) \int_{0}^{s} d s^{\prime} e^{Q \Lambda(\mathbf{k}, \mathbf{c})\left(s-s^{\prime}\right)} Q \tilde{R}\left(\mathbf{k}, \mathbf{c}, s^{\prime}\right) \\
& =-\xi_{2 \perp}(\mathbf{c}) \Pi(\mathbf{k}, s),
\end{aligned}
$$

where we have used the definitions of $S(\mathbf{k}, s)$ and the fluctuating pressure tensor, $\Pi(\mathbf{k}, s)$, Eqs. (6.12) and (6.13). Finally, by multiplying Eq. (B1) by $\hat{\mathbf{k}}_{\perp} \cdot \mathbf{c}$ and further integrating over velocities, we obtain the equation of the transverse velocity of the main text.

\section{Autocorrelation function of $R_{w}(\mathbf{k}, s)$}

In this Appendix we evaluate the correlation function of the noise of the transverse velocity field, $R_{w}(\mathbf{k}, s)$. We consider $\mathbf{k} \neq \mathbf{0}, s_{1}<s_{2}$ with $s_{1}$ large. It is convenient to introduce the following notation for the transverse and parallel components of the vector $\mathbf{c}$

$$
\hat{\mathbf{k}}_{\perp} \cdot \mathbf{c}=c_{\perp}, \quad \hat{\mathbf{k}} \cdot \mathbf{c}=c_{\|} .
$$

The autocorrelation function of $R_{w}(\mathbf{k}, s)$ reads, in terms of $S(\mathbf{k}, s)$ and $\Pi(\mathbf{k}, s)$,

$$
\begin{aligned}
\left\langle R_{w}\left(\mathbf{k}, s_{1}\right) R_{w}\left(-\mathbf{k}, s_{2}\right)\right\rangle_{H} & =\left\langle\left(S\left(\mathbf{k}, s_{1}\right)+\Pi\left(\mathbf{k}, s_{1}\right)\right)\left(S\left(-\mathbf{k}, s_{2}\right)+\Pi\left(-\mathbf{k}, s_{2}\right)\right)\right\rangle_{H} \\
& =\left\langle S\left(\mathbf{k}, s_{1}\right) S\left(-\mathbf{k}, s_{2}\right)\right\rangle_{H}+\left\langle S\left(\mathbf{k}, s_{1}\right) \Pi\left(-\mathbf{k}, s_{2}\right)\right\rangle_{H} \\
& +\left\langle\Pi\left(\mathbf{k}, s_{1}\right) S\left(-\mathbf{k}, s_{2}\right)\right\rangle_{H}+\left\langle\Pi\left(\mathbf{k}, s_{1}\right) \Pi\left(-\mathbf{k}, s_{2}\right)\right\rangle_{H}
\end{aligned}
$$

We now calculate each correlation function taking into account the microscopic expressions of $S(\mathbf{k}, s)$ and $\Pi(\mathbf{k}, s)$, Eqs. (6.12) and (6.13), and the correlation function of the noise of the Boltzmann-Langevin equation, Eqs. (4.21) and (4.22). 
The first term is

$$
\begin{aligned}
\left\langle S\left(\mathbf{k}, s_{1}\right) S\left(-\mathbf{k}, s_{2}\right)\right\rangle_{H} & =\int d \mathbf{c}_{1} \int d \mathbf{c}_{2} c_{1 \perp} c_{2 \perp}\left\langle\tilde{R}\left(\mathbf{k}, \mathbf{c}_{1}, s_{1}\right) \tilde{R}\left(-\mathbf{k}, \mathbf{c}_{2}, s_{2}\right)\right\rangle_{H} \\
& =\frac{\tilde{V}^{2}}{N} \delta\left(s_{1}-s_{2}\right) \int d \mathbf{c}_{1} \int d \mathbf{c}_{2} c_{1 \perp} c_{2 \perp} \Gamma\left(\mathbf{c}_{1}, \mathbf{c}_{2}\right) \\
& =\frac{\tilde{V}^{2}}{N} \delta\left(s_{1}-s_{2}\right) \int d \mathbf{c}_{1} \int d \mathbf{c}_{2} c_{1 \perp} c_{2 \perp} \bar{T}\left(\mathbf{c}_{1}, \mathbf{c}_{2}\right) \chi_{H}\left(c_{1}\right) \chi_{H}\left(c_{2}\right) \\
& =\xi^{2} \frac{\tilde{V}^{2}}{N} \delta\left(s_{1}-s_{2}\right) .
\end{aligned}
$$

where we have used the relation $\int d \mathbf{c}_{1} \int d \mathbf{c}_{2} c_{1 \perp} c_{2 \perp} \bar{T}\left(\mathbf{c}_{1}, \mathbf{c}_{2}\right) \chi_{H}\left(c_{1}\right) \chi_{H}\left(c_{2}\right)=\tilde{\xi}^{2}$, that is proved in [14].

The second correlation function is

$$
\begin{array}{rl}
\left\langle S\left(\mathbf{k}, s_{1}\right)\right. & \left.\Pi\left(-\mathbf{k}, s_{2}\right)\right\rangle_{H} \\
=\int d \mathbf{c}_{1} c_{1 \perp} i k \int_{0}^{s_{2}} d & d s \int d \mathbf{c}_{2} c_{2 \|} c_{2 \perp} e^{Q_{2} \Lambda\left(-\mathbf{k}, \mathbf{c}_{2}\right)\left(s_{2}-s\right)}\left\langle\tilde{R}\left(\mathbf{k}, \mathbf{c}_{1}, s_{1}\right) Q_{2} \tilde{R}\left(-\mathbf{k}, \mathbf{c}_{2}, s\right)\right\rangle_{H} \\
& =i k \frac{\tilde{V}^{2}}{N} \int d \mathbf{c}_{1} \int d \mathbf{c}_{2} c_{1 \perp} c_{2 \|} c_{2 \perp} e^{Q_{2} \Lambda\left(-\mathbf{k}, \mathbf{c}_{2}\right)\left(s_{2}-s_{1}\right)} Q_{2} \Gamma\left(\mathbf{c}_{1}, \mathbf{c}_{2}\right) .
\end{array}
$$

Here, we have changed the sign of $\Pi(-\mathbf{k}, s)$ because we are dealing with the $-\mathbf{k}$ component and then $\hat{\mathbf{k}} \rightarrow-\hat{\mathbf{k}}$ (we do not change $\hat{\mathbf{k}}_{\perp} \rightarrow-\hat{\mathbf{k}}_{\perp}$, because this vector comes from the projector $P$ and it is fixed).

The third term vanishes

$$
\begin{aligned}
\left\langle\Pi\left(\mathbf{k}, s_{1}\right) S\left(-\mathbf{k}, s_{2}\right)\right\rangle_{H} & =-i k \int_{o}^{s_{1}} d s^{\prime} \int d \mathbf{c}_{1} c_{1 \|} c_{1 \perp} \int d \mathbf{c}_{2} c_{2 \perp} \\
& \times e^{Q_{1} \Lambda\left(\mathbf{k}, \mathbf{c}_{1}\right)\left(s-s^{\prime}\right)} Q_{1}\left\langle\tilde{R}\left(\mathbf{k}, \mathbf{c}_{1}, s^{\prime}\right) \tilde{R}\left(-\mathbf{k}, \mathbf{c}_{2}, s_{2}\right)\right\rangle_{H}=0
\end{aligned}
$$

because $\left\langle\tilde{R}\left(s^{\prime}\right) \tilde{R}\left(s_{2}\right)\right\rangle_{H}=0$ for $s^{\prime} \in\left(0, s_{1}\right)$ with $s_{1}<s_{2}$.

Finally, we evaluate the last term to second order in $k$

$$
\begin{aligned}
& \left\langle\Pi\left(\mathbf{k}, s_{1}\right) \Pi\left(-\mathbf{k}, s_{2}\right)\right\rangle_{H} \\
& \simeq k^{2} \int_{0}^{s_{1}} d s_{1}^{\prime} \int_{0}^{s_{2}} d s_{2}^{\prime} \int d \mathbf{c}_{1} \int d \mathbf{c}_{2} c_{1 \|} c_{1 \perp} c_{2 \|} c_{2 \perp} e^{\Lambda\left(\mathbf{c}_{1}\right)\left(s_{1}-s_{1}^{\prime}\right)+\Lambda\left(\mathbf{c}_{2}\right)\left(s_{2}-s_{2}^{\prime}\right)} \\
& \times\left\langle\tilde{R}\left(\mathbf{k}, \mathbf{c}_{1}, s_{1}^{\prime}\right) \tilde{R}\left(-\mathbf{k}, \mathbf{c}_{2}, s_{2}^{\prime}\right)\right\rangle_{H} \\
& =\frac{\tilde{V}^{2}}{N} k^{2} \int_{0}^{s_{1}} d s_{1}^{\prime} \int_{0}^{s_{2}} d s_{2}^{\prime} \int d \mathbf{c}_{1} \int d \mathbf{c}_{2} c_{1 \|} c_{1 \perp} c_{2 \|} c_{2 \perp} e^{\Lambda\left(\mathbf{c}_{1}\right)\left(s_{1}-s_{1}^{\prime}\right)+\Lambda\left(\mathbf{c}_{2}\right)\left(s_{2}-s_{2}^{\prime}\right)} \Gamma\left(\mathbf{c}_{1}, \mathbf{c}_{2}\right) \delta\left(s_{1}^{\prime}-s_{2}^{\prime}\right)
\end{aligned}
$$




$$
\begin{aligned}
& =\frac{\tilde{V}^{2}}{N} k^{2} \int d \mathbf{c}_{1} \int d \mathbf{c}_{2} c_{1 \|} c_{1 \perp} c_{2 \|} c_{2 \perp} \int_{0}^{s_{1}} d s e^{\Lambda\left(\mathbf{c}_{1}\right)\left(s_{1}-s\right)+\Lambda\left(\mathbf{c}_{2}\right)\left(s_{2}-s\right)} \Gamma\left(\mathbf{c}_{1}, \mathbf{c}_{2}\right) \\
& =\frac{\tilde{V}^{2}}{N} k^{2} \int d \mathbf{c}_{1} \int d \mathbf{c}_{2} c_{1 \|} c_{1 \perp} c_{2 \|} c_{2 \perp} e^{\Lambda\left(\mathbf{c}_{1}\right) s_{1}+\Lambda\left(\mathbf{c}_{2}\right) s_{2}} \int_{0}^{s_{1}} d s e^{-s\left[\Lambda\left(\mathbf{c}_{1}\right)+\Lambda\left(\mathbf{c}_{2}\right)\right]} \Gamma\left(\mathbf{c}_{1}, \mathbf{c}_{2}\right) \\
& \simeq \frac{\tilde{V}^{2}}{N} k^{2} \int d \mathbf{c}_{1} \int d \mathbf{c}_{2} c_{1 \|} c_{1 \perp} c_{2 \|} c_{2 \perp} e^{\Lambda\left(\mathbf{c}_{2}\right)\left(s_{2}-s_{1}\right)} \\
& \times\left[\Lambda\left(\mathbf{c}_{1}\right)+\Lambda\left(\mathbf{c}_{2}\right)\right]^{-1}\left[-\Gamma\left(\mathbf{c}_{1}, \mathbf{c}_{2}\right)+\xi^{2} \frac{\partial}{\partial \mathbf{c}_{1}} \cdot \frac{\partial}{\partial \mathbf{c}_{2}} \chi_{H}\left(c_{1}\right) \chi_{H}\left(c_{2}\right)\right]
\end{aligned}
$$

where, in the last step, we have taken into account that $s_{1}$ is large and we have introduced the term $\frac{\partial}{\partial \mathbf{c}_{1}} \cdot \frac{\partial}{\partial \mathbf{c}_{2}} \chi_{H}\left(c_{1}\right) \chi_{H}\left(c_{2}\right)$. This term does not contribute to the integral, but is written for convenience, to make a connection with the global correlation function $\phi_{H}\left(\mathbf{c}_{1}, \mathbf{c}_{2}\right) \equiv \int d \mathbf{l} h_{H}\left(\mathbf{l}, \mathbf{c}_{1}, \mathbf{c}_{2}\right)$ which fulfils Eq. (6.18). In doing so, we find that the autocorrelation function of $\Pi(\mathbf{k}, s)$ reads

$$
\left\langle\Pi\left(\mathbf{k}, s_{1}\right) \Pi\left(-\mathbf{k}, s_{2}\right)\right\rangle_{H} \simeq \frac{\tilde{V}^{2}}{N} k^{2} C_{x y}\left(s_{2}-s_{1}\right),
$$

where

$$
C_{x y}\left(s_{2}-s_{1}\right)=\int d \mathbf{c}_{1} \int d \mathbf{c}_{2} c_{1 x} c_{1 y} c_{2 x} c_{2 y} e^{\Lambda_{2}\left(s_{2}-s_{1}\right)} \phi_{H}\left(\mathbf{c}_{1}, \mathbf{c}_{2}\right) .
$$

As $\phi_{H}\left(\mathbf{c}_{1}, \mathbf{c}_{2}\right)$ is the integral of the correlation function $h_{H}\left(\mathbf{l}, \mathbf{c}_{1}, \mathbf{c}_{2}\right), C_{x y}$ can be identified as the correlation function of the global quantity $\sum_{i=1}^{N} V_{x}(t) V_{y}(t)$.

\section{Evaluation of $\langle S(\mathbf{k}, 0) \Pi(-\mathbf{k}, s)\rangle_{H}$}

In this Appendix we evaluate the time integral of the correlation function $\langle S(\mathbf{k}, 0) \Pi(-\mathbf{k}, s)\rangle_{H}$ in the hydrodynamic limit. Using the notation of the previous Appendix, we have

$$
\begin{aligned}
& \int_{0}^{\infty} d s\langle S(\mathbf{k}, 0) \Pi(-\mathbf{k}, s)\rangle_{H} \\
& =i k \frac{\tilde{V}^{2}}{N} \int d \mathbf{c}_{1} \int d \mathbf{c}_{2} c_{1 \perp} c_{2 \|} c_{2 \perp}\left[\frac{e^{Q_{2} \Lambda\left(-\mathbf{k}, \mathbf{c}_{2}\right) s}}{Q_{2} \Lambda\left(-\mathbf{k}, \mathbf{c}_{2}\right)}\right]_{0}^{\infty} Q_{2} \Gamma\left(\mathbf{c}_{1}, \mathbf{c}_{2}\right) \\
& =-i k \frac{\tilde{V}^{2}}{N} \int d \mathbf{c}_{1} \int d \mathbf{c}_{2} c_{1 \perp} c_{2 \|} c_{2 \perp} \frac{1}{Q_{2} \Lambda\left(-\mathbf{k}, \mathbf{c}_{2}\right)} Q_{2} \Gamma\left(\mathbf{c}_{1}, \mathbf{c}_{2}\right) \\
& =i k \frac{\tilde{V}^{2}}{N} \int d \mathbf{c}_{1} \int d \mathbf{c}_{2} c_{1 \perp} c_{2 \|} c_{2 \perp} \frac{Q_{2}\left[\Lambda\left(\mathbf{k}, \mathbf{c}_{1}\right)+\Lambda\left(-\mathbf{k}, \mathbf{c}_{2}\right)\right]}{Q_{2} \Lambda\left(-\mathbf{k}, \mathbf{c}_{2}\right)} \phi_{H}\left(\mathbf{k}, \mathbf{c}_{1}, \mathbf{c}_{2}\right),
\end{aligned}
$$

where we have introduced the function

$$
\phi_{H}\left(\mathbf{k}, \mathbf{c}_{1}, \mathbf{c}_{2}\right)=\int d \mathbf{l} e^{-i \mathbf{k} \cdot \mathbf{l}} h_{H}\left(\mathbf{l}, \mathbf{c}_{1}, \mathbf{c}_{2}\right),
$$


that fulfils the Fourier transform of Eq. (2.41)

$$
\left[\Lambda\left(\mathbf{k}, \mathbf{c}_{1}\right)+\Lambda\left(-\mathbf{k}, \mathbf{c}_{2}\right)\right] \phi_{H}\left(\mathbf{k}, \mathbf{c}_{1}, \mathbf{c}_{2}\right)=-\Gamma\left(\mathbf{c}_{1}, \mathbf{c}_{2}\right)+\xi^{2} \frac{\partial}{\partial \mathbf{c}_{1}} \cdot \frac{\partial}{\partial \mathbf{c}_{2}} \chi_{H}\left(c_{1}\right) \chi_{H}\left(c_{2}\right) \delta(\mathbf{k}) .
$$

Note that the last term in the previous equation only appears for $\mathbf{k}=\mathbf{0}$. Taking into account that $c_{\perp} \chi_{H}(c)$ is left eigenfunction associated with the null eigenvalue and after some algebra we obtain

$$
\begin{aligned}
& \int_{0}^{\infty} d s\langle S(\mathbf{k}, 0) \Pi(-\mathbf{k}, s)\rangle_{H} \\
& =\frac{\tilde{V}^{2}}{N} k^{2} \int d \mathbf{c}_{1} \int d \mathbf{c}_{2} c_{1 \|} c_{1 \perp} c_{2 \|} c_{2 \perp} \frac{1}{Q_{2} \Lambda\left(-\mathbf{k}, \mathbf{c}_{2}\right)} \phi_{H}\left(\mathbf{k}, \mathbf{c}_{1}, \mathbf{c}_{2}\right) \\
& +\frac{\tilde{V}^{2}}{N} i k \int d \mathbf{c}_{1} \int d \mathbf{c}_{2} c_{1 \perp} c_{2 \|} c_{2 \perp} \phi_{H}\left(\mathbf{k}, \mathbf{c}_{1}, \mathbf{c}_{2}\right) .
\end{aligned}
$$

Now, let us consider the hydrodynamic limit of (D4). The first term gives

$$
\frac{\tilde{V}^{2}}{N} k^{2} \int d \mathbf{c}_{1} \int d \mathbf{c}_{2} c_{1 \|} c_{1 \perp} c_{2 \|} c_{2 \perp} \frac{1}{Q_{2} \Lambda\left(-\mathbf{k}, \mathbf{c}_{2}\right)} \phi_{H}\left(\mathbf{k}, \mathbf{c}_{1}, \mathbf{c}_{2}\right) \rightarrow-\frac{\tilde{V}^{2}}{N} k^{2} \int_{0}^{\infty} d s C_{x y}(s) .
$$

The second term can be evaluated by using the following expansion in powers of $k$

$$
[\Lambda(\mathbf{c})-i \mathbf{k} \cdot \mathbf{c}]^{-1} \simeq \Lambda(\mathbf{c})^{-1}+\Lambda(\mathbf{c})^{-1}(i \mathbf{k} \cdot \mathbf{c}) \Lambda(\mathbf{c})^{-1},
$$

which yields

$$
\begin{array}{r}
\frac{\tilde{V}^{2}}{N} i k \int d \mathbf{c}_{1} \int d \mathbf{c}_{2} c_{1 \perp} c_{2 \|} c_{2 \perp} \phi_{H}\left(\mathbf{k}, \mathbf{c}_{1}, \mathbf{c}_{2}\right) \\
\rightarrow \frac{\tilde{V}^{2}}{N} k^{2} \int_{0}^{\infty} d s C_{x y}(s)-\frac{\tilde{V}^{2}}{N} k^{2} \int d \mathbf{c}_{1} \int d \mathbf{c}_{2} c_{1 x} c_{2 x} c_{2 y} \int_{0}^{\infty} d s e^{\Lambda\left(\mathbf{c}_{2}\right) s} c_{2 y} \phi_{H}\left(\mathbf{c}_{1}, \mathbf{c}_{2}\right) .
\end{array}
$$

Taking into account (D5) and (D7), we obtain

$$
\begin{aligned}
\int_{0}^{\infty} d s\langle S(\mathbf{k}, 0) \Pi(-\mathbf{k}, s)\rangle & \rightarrow-\frac{\tilde{V}^{2}}{N} k^{2} \int d \mathbf{c}_{1} \int d \mathbf{c}_{2} c_{1 x} c_{2 x} c_{2 y} \int_{0}^{\infty} d s e^{\Lambda\left(\mathbf{c}_{2}\right) s} c_{2 y} \phi_{H}\left(\mathbf{c}_{1}, \mathbf{c}_{2}\right) \\
& =\frac{\tilde{V}^{2}}{N} k^{2} \int d \mathbf{c}_{1} \int d \mathbf{c}_{2} c_{1 x} c_{2 x} c_{2 y} \Lambda\left(\mathbf{c}_{2}\right)^{-1} c_{2 y} \phi_{H}\left(\mathbf{c}_{1}, \mathbf{c}_{2}\right)
\end{aligned}
$$

\section{E Evaluation of the $k^{2}$ contribution of $\left\langle R_{w}\left(\mathbf{k}, s_{1}\right) R_{w}\left(-\mathbf{k}, s_{2}\right)\right\rangle_{H}$}

In this Appendix we evaluate the $k^{2}$ component of the correlation function of $R_{w}$ in terms of the one- and two-particle distribution functions, $\chi_{H}$ and $\chi_{2}$

$$
\phi_{H}\left(\mathbf{c}_{1}, \mathbf{c}_{2}\right)=\chi_{H}\left(c_{1}\right) \delta\left(\mathbf{c}_{12}\right)+\chi_{2}\left(\mathbf{c}_{1}, \mathbf{c}_{2}\right) .
$$


The first term is

$$
\begin{aligned}
\int_{0}^{\infty} d s C_{x y}(s) & =\int_{0}^{\infty} d s \int d \mathbf{c}_{1} \int d \mathbf{c}_{2} c_{1 x} c_{1 y} c_{2 x} c_{2 y} e^{s \Lambda\left(\mathbf{c}_{2}\right)} \phi_{H}\left(\mathbf{c}_{1}, \mathbf{c}_{2}\right) \\
& =-\int d \mathbf{c}_{1} \int d \mathbf{c}_{2} c_{1 x} c_{1 y} c_{2 x} c_{2 y} \Lambda\left(\mathbf{c}_{2}\right)^{-1} \phi_{H}\left(\mathbf{c}_{1}, \mathbf{c}_{2}\right) \\
& =-\int d \mathbf{c} c_{x} c_{y} \Lambda(\mathbf{c})^{-1} c_{x} c_{y} \chi_{H}(c) \\
& -\int d \mathbf{c}_{1} \int d \mathbf{c}_{2} c_{1 x} c_{1 y} c_{2 x} c_{2 y} \Lambda\left(\mathbf{c}_{2}\right)^{-1} \chi_{2}\left(\mathbf{c}_{1}, \mathbf{c}_{2}\right) .
\end{aligned}
$$

If we do the same in the second term, we have

$$
\begin{aligned}
\int d \mathbf{c}_{1} \int d \mathbf{c}_{2} c_{1 x} c_{2 x} c_{2 y} \Lambda\left(\mathbf{c}_{2}\right)^{-1} c_{2 y} \phi_{H}\left(\mathbf{c}_{1}, \mathbf{c}_{2}\right) & =\int d \mathbf{c} c_{x} c_{y} \Lambda(\mathbf{c})^{-1} c_{x} c_{y} \chi_{H}(c) \\
& +\int d \mathbf{c}_{1} \int d \mathbf{c}_{2} c_{1 x} c_{2 x} c_{2 y} \Lambda\left(\mathbf{c}_{2}\right)^{-1} c_{2 y} \chi_{2}\left(\mathbf{c}_{1}, \mathbf{c}_{2}\right) .
\end{aligned}
$$

It can be seen that the sum of the two terms only depends on the two-particle correlation function and we obtain the $k^{2}$ part of Eq. (6.21).

\section{References}

[1] A. Baskaran, J. W. Dufty, J. J. Brey. Transport coefficients for the hard-sphere granular fluid. Phys. Rev. E, 77 (2008), No. 3, 031311.

[2] M. Bixon, R. Zwanzig. Boltzmann-Langevin Equation and Hydrodynamic Fluctuations. Phys. Rev., 187 (1969), No. 1, 267-272.

[3] J. J. Brey, J. W. Dufty, M. J. Ruiz-Montero, in Granular Gas Dynamics, edited by T. Pöschel and N. Brilliantov. Springer, Berlin, 2003.

[4] J. J. Brey, M. I. García de Soria, P. Maynar. Breakdown of hydrodynamics in the inelastic Maxwell model of granular gases. Phys. Rev. E, 82 (2010), No. 2, 021303.

[5] J. J. Brey, M. I. García de Soria, P. Maynar. Breakdown of the fluctuation-dissipation relations in granular gases. EPL, 84 (2008), No. 2, 24002.

[6] J. J. Brey, P. Maynar, M. I. García de Soria. Fluctuating hydrodynamics for dilute granular gases. Phys. Rev. E, 79 (2009), No. 5, 051305.

[7] J. J. Brey, M. I. García de Soria, P. Maynar, M. J. Ruiz-Montero. Energy fluctuations in the homogeneous cooling state of granular gases. Phys. Rev. E, 70 (2004), No. 1, 011302. 
[8] J. J. Brey, M. J. Ruiz-Montero, F. Moreno. Boundary conditions and normal state for a vibrated granular fluid. Phys. Rev. E, 62 (2000), No. 4, 5339-5346.

[9] R. Cafiero, S. Luding, H. J. Herrmann. Two-Dimensional Granular Gas of Inelastic Spheres with Multiplicative Driving. Phys. Rev. Lett., 84 (2000), No. 26, 6014-6017.

[10] M. H. Ernst, E. Trizac, A. Barrat. The Boltzmann Equation for Driven Systems of Inelastic Soft Spheres. J. Stat. Phys., 124 (2006), No. 2-4, 549-586.

[11] M. H. Ernst, E. Trizac, A. Barrat. The rich behavior of the Boltzmann equation for dissipative gases. Europhys. Lett., 76 (2006), No. 1, 56-62.

[12] A. Fiege, T. Aspelmeier, A. Zippelius. Long-Time Tails and Cage Effect in Driven Granular Fluids. Phys. Rev. Lett., 102 (2009), No. 9, 098001.

[13] M. I. García de Soria, P. Maynar, G. Schehr, A. Barrat, E. Trizac. Dynamics of annihilation. II. Fluctuations of global quantities. Phys. Rev. E, 77 (2008), No. 5, 051128.

[14] M. I. García de Soria, P. Maynar, E. Trizac. Energy fluctuations in a randomly driven granular fluid. Mol. Phys., 107 (2009), No. 4-6, 383-392.

[15] V. Garzó, J. M. Montanero. Transport coefficients of a heated granular gas. Physica A, 313 (2002), No. 3-4, 336-356.

[16] I. Goldhirsch, G. Zanetti. Clustering instability in dissipative gases. Phys. Rev. Lett., 70 (1993), No. 11, 1619-1622.

[17] A. Goldshtein, M. Shapiro. Mechanics of collisional motion of granular materials. Part 1. General hydrodynamic equations. J. Fluid. Mech., 282 (1995), 75-114.

[18] P. K. Haff. Grain flow as a fluid-mechanical phenomenon. J. Fluid. Mech., 134 (1983), 401430.

[19] C. C. Maaß, N. Isert, G. Maret, C. M. Aegerter. Experimental Investigation of the Freely Cooling Granular Gas. Phys. Rev. Lett., 100 (2008), No. 24, 248001.

[20] P. Maynar, M. I. García de Soria, E. Trizac. Fluctuating hydrodynamics for driven granular gases. EPJ ST, 179 (2009), 123-139.

[21] S. McNamara, W. R. Young. Dynamics of a freely evolving, two-dimensional granular medium. Phys. Rev. E, 53 (1996), No. 5, 5089-5100.

[22] J. M. Montanero, A. Santos. Computer simulation of uniformly heated granular fluids. Granular Matter, 2 (2000), No. 2, 53-64.

[23] S. J. Moon, M. D. Shattuck, J. B. Swift. Velocity distributions and correlations in homogeneously heated granular media. Phys. Rev E, 64 (2001), No. 3, 031303. 
[24] I. Pagonabarraga, E. Trizac, T. P. C. van Noije, M. H. Ernst. Randomly driven granular fluids: Collisional statistics and short scale structure. Phys. Rev. E, 65 (2001), No. 1, 011303.

[25] B. Painter, M. Dutt, R. Behringer. Energy dissipation and clustering for a cooling granular material on a substrate. Physica D, 175 (2003), No. 1-2, 43-68.

[26] A. Prevost, D. A. Egolf, J. S. Urbach. Forcing and Velocity Correlations in a Vibrated Granular Monolayer. Phys. Rev. Lett., 89 (2002), No. 8, 084301.

[27] A. Puglisi, V. Loreto, U. M. B. Marconi, A. Vulpiani. Kinetic approach to granular gases. Phys. Rev E, 59 (1999), No. 5, 5582-5595.

[28] P. Résibois, M. de Leener. Classical Kinetic Theory of Fluids. Wiley, New York, 1977.

[29] N. G. van Kampen. Stochastic Proccesses in Physics and Chemistry. North-Holland, Amsterdam, 1992.

[30] T. P. C. van Noije, M. H. Ernst. Velocity distributions in homogeneous granular fluids: the free and the heated case. Granular Matter, 1 (1998), No. 2, 57-64.

[31] T. P. C. van Noije, M. H. Ernst, E. Trizac, I. Pagonabarraga. Randomly driven granular fluids: Large-scale structure. Phys. Rev. E, 59 (1999), No. 4, 4326-4341.

[32] P. Visco, A. Puglisi, A. Barrat, F. van Wijland, E. Trizac. Energy fluctuations in vibrated and driven granular gases. Eur. Phys. J. B, 51 (2006), No. 3, 377-387.

[33] D. R. M. Williams, F. C. MacKintosh. Driven granular media in one dimension: Correlations and equation of state. Phys. Rev. E, 54 (1996), No. 1, R9-R12. 